\title{
A spectroscopic study of the open cluster NGC 6250
}

\author{
A. J. Martin, ${ }^{1,2 \star} \dagger$ M. J. Stift,${ }^{1}$ L. Fossati, ${ }^{3}$ S. Bagnulo, ${ }^{1 \star}$ C. Scalia, ${ }^{4,5}$ F. Leone ${ }^{4,5}$ \\ and B. Smalley ${ }^{2}$ \\ ${ }^{1}$ Armagh Observatory \& Planetarium, College Hill, Armagh BT61 9DG, UK \\ ${ }^{2}$ Astrophysics Group, Keele University, Staffordshire ST5 5BG, UK \\ ${ }^{3}$ Space Research Institute, Austrian Academy of Sciences, Schmiedlstrasse 6, A-8042 Graz, Austria \\ ${ }^{4}$ Università di Catania, Dipartimento di Fisica e Astronomia, Sezione Astrofisica, Via S. Sofia 78, I-95123 Catania, Italy \\ ${ }^{5}$ INAF-Osservatorio Astrofisico di Catania, Via S. Sofia 78, I-95123 Catania, Italy
}

Accepted 2016 November 22. Received 2016 November 22; in original form 2016 June 10

\begin{abstract}
We present the chemical abundance analysis of 19 upper main-sequence stars of the young open cluster NGC $6250(\log t \sim 7.42 \mathrm{yr})$. This work is part of a project aimed at setting observational constraints on the theory of atomic diffusion in stellar photospheres, by means of a systematic study of the abundances of the chemical elements of early F-, A- and late B-type stars of well-determined age. Our data set consists of low-, medium- and high-resolution spectra obtained with the Fibre Large Array Multi Element Spectrograph (FLAMES) instrument of the ESO Very Large Telescope (VLT). To perform our analysis, we have developed a new suite of software tools for the chemical abundance analysis of stellar photospheres in local thermodynamical equilibrium. Together with the chemical composition of the stellar photospheres, we have provided new estimates of the cluster mean radial velocity, proper motion, refined the cluster membership, and we have given the stellar parameters including masses and fractional age. We find no evidence of statistically significant correlation between any of the parameters, including abundance and cluster age, except perhaps for an increase in $\mathrm{Ba}$ abundance with cluster age. We have proven that our new software tool may be successfully used for the chemical abundance analysis of large data sets of stellar spectra.
\end{abstract}

Key words: stars: abundances - open clusters and associations: individual: NGC 6250.

\section{INTRODUCTION}

The spectra of early F-, A- and late B-type stars frequently show a wealth of signatures of various physical phenomena of comparable magnitude, such as, for instance, pulsation, the presence of a magnetic field and a non-homogeneous distribution of the chemical elements (e.g. Landstreet 2004). The latter is an effect of the diffusion of the chemical elements, a mechanism that is particularly important to study because it affects the apparent chemical composition of stars. In principle, effects of the diffusion that operate at a large time-scale (i.e. comparable to the stellar lifetime) may even mimic those due to the Galactic chemical evolution. Therefore, it is important to understand whether the relative chemical composition of the photosphere appear systematically different from that of younger stars.

In order to obtain information about time-dependent processes acting in stellar photospheres, we have chosen to study stars that are member of open clusters of various ages. This is because the

\footnotetext{
^E-mail: ajm@arm.ac.uk (AJM); sba@arm.ac.uk (SB)

$\dagger$ Present address: Armagh Observatory, Planetarium, College Hill, Armagh BT61 9DG, UK
}

age of an open cluster may be determined with a much better accuracy than that of individual stars in the field, in particular when the star is in the first-half of its main-sequence lifetime (e.g. Bagnulo et al. 2006). A second advantage is that open cluster stars are presumably formed with the same chemical composition, so that any difference in the observed chemical composition between cluster members may be directly linked to one of the stellar properties (e.g. effective temperature and/or rotation).

We have considered the low- and mid-resolution spectra of 32 stars observed with the Fibre Large Array Multi Element Spectrograph (FLAMES) instrument of the ESO Very Large Telescope (VLT) in the field of view of the open cluster NGC 6250, and we performed a detailed chemical abundance analysis of the 19 member stars. Our observations are part of a data set containing the spectra of approximately 1000 stars observed as part of a larger effort to explore how various physical effects change as a function of stellar age, in particular to set observational constraints to the theory of atomic diffusion in stellar photospheres (Michaud 1970), both in the cases of magnetic and non-magnetic atmospheres. The overall project includes data for potential members of various open clusters, covering ages from $\log t=6.8$ to 8.9 and distance moduli from 6.4 to 11.8 . The full list of observed open clusters is given by Fossati et al. (2008a). 
The analysis of three of these clusters has been performed by Kilıçoğlu et al. (2016) (NGC6405); Fossati et al. (2007, 2008b, 2010) and Fossati et al. (2011a) (Praesape cluster and NGC 5460). Kilıçoğlu et al. (2016) found NGC 6405 to have an age of $\log t \sim 7.88$, a distance of $400 \mathrm{pc} \pm 50 \mathrm{pc}$ and an $[\mathrm{Fe} / \mathrm{H}]$ metallicity of $0.07 \pm 0.03$. The Praesape cluster has an age of $\log t \sim 8.85 \pm$ 0.15 (González-García et al. 2006) and it is at a distance of $180 \mathrm{pc} \pm$ 10 pc (Robichon et al. 1999). Fossati et al. (2011a) found NGC 5460 to have an age of $\log t \sim 8.2 \pm 0.1$, a distance of $720 \mathrm{pc} \pm 50 \mathrm{pc}$ and a near solar metallicity.

With an age of $\log t \sim 7.42 \mathrm{yr}$ and a distance of $865 \mathrm{pc}$ (Kharchenko et al. 2013), NGC 6250 is both the youngest and most distant cluster analysed as part of this project so far. Further studies completed by different groups, with data that can be used as part of this study, include those by Gebran \& Monier (2008) and Gebran, Monier \& Richard (2008); Gebran et al. (2010) (Coma Berenices, $\log t=8.65$; the Pleiades, $\log t=8.13$; and Hyades, $\log t=8.9$ ); Folsom et al. (2007) and Villanova, Carraro \& Saviane (2009) (NGC 6475, $\log t=8.48$ ); and Stütz et al. (2006) (IC 2391, $\log t=7.66)$.

The study by Bailey, Landstreet \& Bagnulo (2014) searched for trends between chemical abundance and stellar parameters of chemically peculiar Ap stars, to determine whether chemical peculiarities change as a star evolves. This data will allow us to compare the behaviour of chemically peculiar magnetic stars with our sample of chemically normal stars.

To analyse the remaining clusters for this project in a more efficient manner, and in particular to deal with the especially interesting case of magnetic stars, we have developed SPARTI (SpectroPolarimetric Analysis by Radiative Transfer Inversion), a software tool based on the radiative transfer code cossam (Stift 2000; Stift, Leone \& Cowley 2012). SPARTI will be presented in a forthcoming paper (Martin et al., in preparation). In this work, we introduce its simplest version, SPARTI_SIMPLE, specifically designed to deal with the nonmagnetic case. SPARTI_SIMPLE is based around COSSAM_SIMPLE, which in turn is a modified version of the code Cossam for the spectral synthesis of magnetic atmospheres. This approach has the advantage that both magnetic and non-magnetic stars may be analysed in a homogeneous way. Eventually, the comparison of the chemical composition of magnetic and non-magnetic stars belonging to the same cluster will allow us a more accurate analysis of the effects of magnetic fields on the diffusion of the chemical elements in a stellar photosphere. Our new software suite is fully parallelized, which reduces the CPU time required to analyse each star.

In this paper, we first describe COSSAM_SIMPLE and SPARTI_SIMPLE (Sections 2 and 3), then we present the observations (Section 4), we establish cluster membership (Section 5) and we determine the fundamental parameters of the cluster members (Section 6). We then present new spectroscopic observations of the cluster NGC 6250 (Section 4). Finally, we present and discuss our results (Section 7). Our conclusions are summarized in Section 8.

\section{THE COSSAM CODE}

CossAm, the 'Codice per la sintesi spettrale nelle atmosfere magnetiche' is an object-oriented and fully parallelized polarized spectral line synthesis code, under GNU copyleft since the year 2000. It allows the calculation of detailed Stokes IQUV spectra in the Sun and in rotating and/or pulsating stars with dipolar and quadrupolar magnetic geometries. Software archaeology reveals that cossAm harks back to the ALGOL 60 code ANALYSE 65 by Baschek, Holweger $\&$ Traving (1966) and to the FORTRAN code ADRs3 by Chmielewski
(1979). COSSAM is the first code of its kind that takes advantage of the sophisticated concurrent constructs of the ADA programming language that make it singularly easy to parallelize the line synthesis algorithms without having recourse to message passing interfaces. 'Tasks', each of which has its own thread of control and each of which performs a sequence of actions - such as opacity sampling and solving the polarized radiative transfer equation over a given spectral interval - can execute concurrently within the same program on a large number of processor cores. Protected objects, which do not have a thread of control of their own, are accessed in mutual exclusion (i.e. only one process can update a variable at a time) and provide efficient synchronisation with very little overhead.

\subsection{Physics and numerics}

COSSAM assumes a plane-parallel atmosphere and local thermodynamic equilibrium (LTE). It is convenient to use the VALD data base (Piskunov et al. 1995) extracting atomic transition data including radiation damping, Stark broadening and van der Waals broadening constants. The atomic partition functions are calculated with the help of the appropriate routines in ATLAs 12 (Kurucz 2005). Landé factors and $J$-values for the lower and the upper energy levels provided by VALD make it possible to determine the Zeeman splitting and the individual component strengths of each line; in the case the Landé factors are missing, a classical Zeeman triplet is assumed. For the continuous opacity $\kappa_{\mathrm{c}}$ at a given wavelength, Cossam employs ATLAS 12 routines (Kurucz 2005) rewritten in ADA by Bischof (2005). The total line opacities required in the formal solver are determined by full opacity sampling of the $\sigma_{-}, \sigma_{+}$and $\pi$ components separately. The opacity profiles - Voigt and Faraday functions of metallic lines are based on the rational expression found in Hui, Armstrong \& Wray (1978). The approximation to the hydrogen line opacity profiles given in TLUSTY (Hubeny \& Lanz 1995) has proved highly satisfactory and easy to implement. The higher Balmer series members are treated according to Hubeny, Hummer \& Lanz (1994); this recipe is based on the occupation probability formalism (Dappen, Anderson \& Mihalas 1987; Hummer \& Mihalas 1988; Seaton 1990).

By default, cossam employs the Zeeman Feautrier method (Auer, Heasley \& House 1977), reformulated by Alecian \& Stift (2004) in order to treat blends in a static atmosphere. Alternatively, the user can choose the somewhat faster but less accurate DELO method (Rees, Durrant \& Murphy 1989). Since most of the CPU time is spent on opacity sampling, the overall cost of Zeeman Feautrier is only slightly higher compared to DELO. In the local ('solar') case, the emerging Stokes spectrum is calculated for one given point on the solar surface - specified by the position $\mu=\cos \theta=\left(1-r^{2}\right)^{1 / 2}$ - and the attached magnetic vector. In the 'stellar' (disc-integrated) case, COSSAM has to integrate the emerging spectrum over the whole visible hemisphere, i.e. over $0<\mu \leq 1$, taking into account rotation, (non-)radial pulsation and a global dipolar or quadrupolar magnetic field structure.

Different spatial grids are provided to best cater for the different spectral line synthesis problems usually encountered. We may distinguish corotating from observer-centred grids. The former are extensively used in Doppler mapping (see e.g. Vogt, Penrod \& Hatzes 1987); the entire stellar surface is split into elements of approximately equal size. Chemical and/or magnetic spots can easily be modelled with the help of these corotating spatial grids. Observer-centred grids usually are of a fixed type where neither the magnetic field geometry nor rotation and/or pulsation determines the distribution of the quadrature points. Cossam also provides a 
third type of grid, namely an adaptive grid as discussed in Stift (1985) and Fensl (1995). A special algorithm provides optimum 2D-integration by ensuring that the change in the monochromatic opacity matrix between two adjacent quadrature points does not exceed a certain percentage. The point distribution can become very non-uniform, depending on the direction of the magnetic field vector, its azimuth, the Doppler shifts due to rotation and/or pulsation, and on the amount of limb darkening. At the same level of accuracy of the resulting Stokes profiles, it is thus possible to greatly reduce the number of quadrature points compared to fixed grids.

\subsection{COSSAM_SIMPLE, a fast tool for chemical abundance analysis in non-magnetic stars}

In principle, the original version of COSSAM may naturally deal with the non-magnetic case, just by setting the magnetic field strength to zero. Practically, cossam would still perform a number of timeconsuming numerical computations ending into flat-zero Stokes $Q U V$ profiles. Therefore, the original code was modified to take advantage of the various symmetries and simplifications of the nonmagnetic case, and to allow extremely fast, but nevertheless highly accurate integration of intensity profiles even in rapidly rotating stars. COSSAM_SIMPLE calculates local spectra at high wavelength resolution at various positions $\mu=\cos \theta$; the stellar spectrum is then derived by integration over the appropriately shifted local spectra. Instead of the hundreds or even thousands of local spectra to be calculated for the general-purpose 2D-grid, a few dozen local spectra prove sufficient.

After calculating a synthetic spectrum, we convolve it with a Gaussian matching the instrument resolution, the wavelength sampling of the resulting spectrum is then matched to the observed wavelength grid to allow for comparison.

\section{INVERSION METHOD}

SPARTI_SIMPLE is an inversion code that uses COSSAM_SIMPLE to calculate synthetic stellar spectra, and the Levenberg-Marquardt algorithm (LMA) to find the best-fitting parameters to observed stellar spectra. Its free parameters are the chemical abundances of an arbitrary number of chemical elements (including independent abundances for each ionization stage), assuming a fixed model for the stellar atmosphere (hence fixed values of effective stellar temperature $T_{\text {eff }}$ and gravity $\log g$ ).

\subsection{Levenberg-Marquardt algorithm}

The Levenberg-Marquardt algorithm (LMA; Levenberg 1944; Marquardt 1963) is a least-squares technique that combines the GaussNewton and gradient-descent methods. It allows one to determine the minimum of a multivariate function minimizing the expression

$\chi^{2}=\sum_{i=1}^{n} \frac{\left[F_{\bmod }\left(\lambda_{i}, \boldsymbol{x}\right)-F_{\mathrm{obs}}\left(\lambda_{i}\right)\right]^{2}}{\sigma_{i}^{2}}$,

where $F_{\mathrm{obs}}$ is the observed spectrum, $\sigma$ is the error associated with each spectral bin $i, F_{\text {mod }}$ is the synthetic spectrum, convolved with the instrument response, $\boldsymbol{x}$ is the array of free parameters assumed in the spectral synthesis, and $n$ is the number of spectral points.

SPARTI_SIMPLE initially calculates a synthetic spectrum with the chemical abundances set to solar values from Asplund et al. (2009). Initial estimate values of the projected equatorial velocity $v \sin i$, the radial velocity $v_{\text {rad }}$ and the microturbulence $v_{\text {mic }}$ are also required.
After the initial spectrum is generated, the LMA is run and convergence to the best solution is usually reached within four to eight iterations. The algorithm is stopped when the following conditions are met (Aster, Borchers \& Thurber 2013)

$$
\begin{aligned}
& \sum_{i=1}^{n}\left[\nabla \chi^{2}\right]^{2}<\sqrt{\epsilon} \sum_{i=1}^{n}[1+f(i)] \\
& \sum_{i=1}^{n}\left[x(i)-x_{0}(i)\right]^{2}<\sqrt{\epsilon} \sum_{i=1}^{n}\left[1+x(i)^{2}\right] \\
& \sum_{i=1}^{n}\left[F(i)-F_{0}(i)\right]^{2}<\epsilon \sum_{i=1}^{n}\left[1+F(i)^{2}\right],
\end{aligned}
$$

where $x_{0}$ is the previous parameter set, $F_{0}$ is the model spectrum calculated with $x_{0}$ and $\epsilon=10^{-4}$. The maximum number of iterations is set to 50 , if this number is reached, we reassess the starting parameters and rerun SPARTI_SIMPLE.

\subsubsection{Semi-automatic identification of the chemical elements}

To quickly check which elements may be identified in the observed spectrum, after the best fit is found, we re-calculate a number of synthetic spectra, each of which obtained after setting to zero the abundance of a single element. We compare each of these new synthetic spectra with the observed one and we check if the reduced $\chi^{2}$ has varied by more than the signal-to-noise $(\mathrm{S} / \mathrm{N})$ threshold. If there is no change we consider that the element cannot be measured in the observed spectrum. If $\chi^{2}$ has varied, we check the spectrum to determine whether the element has visible spectral lines.

\subsection{Abundance uncertainties}

We estimate the uncertainties of the best-fitting parameters, including $v_{\text {mic }}$ and $v \sin i$ by taking the square root of the diagonal values of the covariance matrix

$\operatorname{cov}(\widehat{\boldsymbol{x}})=s^{2} \Delta \boldsymbol{F} \Delta \boldsymbol{F}^{T}$,

where $\widehat{\boldsymbol{x}}$ is the vector of best fit and

$s=\sqrt{\frac{\sum_{i=0}^{n}\left[F_{\bmod }\left(\lambda_{i}\right)-F_{\mathrm{obs}}\left(\lambda_{i}\right)\right]^{2}}{m-n},}$

where $n$ is the number of wavelength points in the spectrum and $m$ the number of best-fitting parameters.

The error so estimated is only a lower limit since SPARTI_SIMPLE assumes fixed values of $T_{\text {eff }}$ and $\log g$. Therefore, the covariance matrix does not contain information on the effects of the uncertainties of $T_{\text {eff }}$ or $\log g$. To take these uncertainties into account, we run the inversion another four times, setting $T_{\text {eff }}=T_{\text {eff }}^{0} \pm \Delta T_{\text {eff }}$ and $\log g=\log g^{0} \pm \Delta \log g$, where $T_{\text {eff }}^{0}$ and $\log g^{0}$ are our best estimates for $T_{\text {eff }}$ and $\log g$, respectively, and $\Delta T_{\text {eff }}$ and $\Delta \log g$ are their errors. As abundance uncertainty, we finally adopt

$\sigma=\sqrt{\sigma_{\mathrm{cov}}^{2}+\sigma_{T_{\mathrm{eff}}}^{2}+\sigma_{\log g}^{2}}$,

where $\sigma_{\text {cov }}$ is the error values calculated from equation (4), $\sigma_{T_{\text {eff }}}$ is half of the difference between the best-fitting values for the abundances obtained assuming $T_{\text {eff }}=T_{\text {eff }}^{0}+\Delta T_{\text {eff }}$ and $T_{\text {eff }}=T_{\text {eff }}^{0}-$ $\Delta T_{\text {eff }}$, and $\sigma_{\log g}$ half of the difference between the best-fitting values for the abundances obtained assuming $\log g=\log g^{0}+\Delta \log g$ and $\log g=\log g^{0}-\Delta \log g$. 
Table 1. The fundamental parameters associated with the Sun. Calculated both by Prsa et al. (2016) and by the method used in this work.

\begin{tabular}{lcc}
\hline Fundamental parameters & Prsa et al. (2016) & This work \\
\hline$T_{\text {eff }}(\mathrm{K})$ & 5777 & $5800 \pm 200$ \\
$\log g(\mathrm{cgs})$ & 4.438 & $4.49 \pm 0.1$ \\
$v \sin i\left(\mathrm{~km} \mathrm{~s}^{-1}\right)$ & 1.2 & $2.6 \pm 0.1$ \\
$v_{\text {mic }}\left(\mathrm{km} \mathrm{s}^{-1}\right)$ & 0.875 & $1.0 \pm 0.03$ \\
\hline
\end{tabular}

Table 2. The fundamental parameters associated with HD 32115. Calculated both by Fossati et al. (2011b) and by the method used in this work.

\begin{tabular}{lcc}
\hline Fundamental parameters & Fossati et al. (2011b) & This work \\
\hline$T_{\text {eff }}(\mathrm{K})$ & $7250 \pm 100$ & $7300 \pm 200$ \\
$\log g(\mathrm{cgs})$ & $4.2 \pm 0.1$ & $4.2 \pm 0.1$ \\
$v \sin i\left(\mathrm{~km} \mathrm{~s}^{-1}\right)$ & $8.3 \pm 0.5$ & $14.21 \pm 0.07$ \\
$v_{\text {mic }}\left(\mathrm{km} \mathrm{s}^{-1}\right)$ & $2.5 \pm 0.2$ & $2.5 \pm 0.02$ \\
\hline
\end{tabular}

Table 3. The fundamental parameters associated with the $21 \mathrm{Peg}$. Calculated both by Fossati et al. (2009) and by the method used in this work.

\begin{tabular}{lcc}
\hline Fundamental parameters & Fossati et al. (2009) & This work \\
\hline$T_{\text {eff }}(\mathrm{K})$ & $10400 \pm 200$ & $10400 \pm 200$ \\
$\log g(\mathrm{cgs})$ & $3.55 \pm 0.1$ & $3.5 \pm 0.1$ \\
$v \sin i\left(\mathrm{~km} \mathrm{~s}^{-1}\right)$ & $3.76 \pm 0.35$ & $4.2 \pm 0.1$ \\
$v_{\text {rad }}\left(\mathrm{km} \mathrm{s}^{-1}\right)$ & $0.5 \pm 0.5$ & $0.40 \pm 0.04$ \\
$v_{\text {mic }}\left(\mathrm{km} \mathrm{s}^{-1}\right)$ & $0.5 \pm 0.5$ & $0.4 \pm 0.2$ \\
\hline
\end{tabular}

\subsection{Test cases: the Sun, HD 32115 and 21 Peg}

In order to check the full consistency between the results obtained with SPARTI_SIMPLE and those obtained in the previous spectral analysis of open cluster members, we have performed a test spectral analysis of the Sun, HD 32115 and the star 21 Peg. We chose these stars because they represent three different regimes: in the solar spectrum, the Balmer lines are only sensitive to temperature; in the spectrum of HD 32115, the Balmer lines are sensitive to temperature and surface gravity; and in the spectrum of $21 \mathrm{Peg}$, the Balmer lines are more sensitive to surface gravity. We calculate the fundamental parameters for each star shown in Tables $1-3$. Good agreement is seen between the parameters we calculated and the previously published results. The higher value of $v \sin i$ that we measure for the Sun is due to the fact that we do not account for macroturbulence broadening, while the technique used to calculate the value of $v \sin i$ given in Prsa et al. (2016) does. For HD 32115, we derived the $T_{\text {eff }}$ and $\log g$ from the Balmer lines, since Fossati et al. (2011b) showed the ionization balance of $\mathrm{Fe}$ is not sufficient in this star to determine $\log g$ accurately.

The $v_{\text {mac }}$ of the Sun ranges from 1 to $4 \mathrm{~km} \mathrm{~s}^{-1}$; however, we convolved the solar spectrum to a resolution of 25900 to simulate the analysis of our GIRAFFE spectra. At this resolution, the effect of $v_{\text {mac }}$ is not visible in our spectra. The analysis of the chemical abundances of the Sun shows good agreement with the results of Asplund et al. (2009) within the error bars shown in Table 4.

To perform the comparison between the chemical abundances determined by Fossati et al. (2009) and those determined by SPARTI_SIMPLE for $21 \mathrm{Peg}$, we choose the same lines, atomic parameters and ATLAS12 model atmosphere as Fossati et al. (2009) and
Table 4. A comparison between the chemical abundances of the Sun calculated by Asplund et al. (2009) and those calculated by SPARTI_SIMPLE.

\begin{tabular}{lccr}
\hline \multicolumn{4}{c}{$\log (N / \mathrm{H})$} \\
Element & $\begin{array}{c}\text { Asplund et al. } \\
(2009)\end{array}$ & SPARTI_SIMPLE & \multicolumn{1}{c}{$\Delta$} \\
\hline $\mathrm{Mg}$ & $7.60 \pm 0.04$ & $7.63 \pm 0.02$ & -0.03 \\
$\mathrm{Ti}$ & $4.95 \pm 0.05$ & $4.96 \pm 0.01$ & -0.01 \\
$\mathrm{~V}$ & $3.93 \pm 0.08$ & $3.97 \pm 0.01$ & -0.04 \\
$\mathrm{Cr}$ & $5.64 \pm 0.04$ & $5.60 \pm 0.01$ & 0.04 \\
$\mathrm{Fe}$ & $7.50 \pm 0.04$ & $7.49 \pm 0.01$ & 0.01 \\
$\mathrm{Ni}$ & $6.22 \pm 0.04$ & $6.21 \pm 0.01$ & 0.01 \\
\hline
\end{tabular}

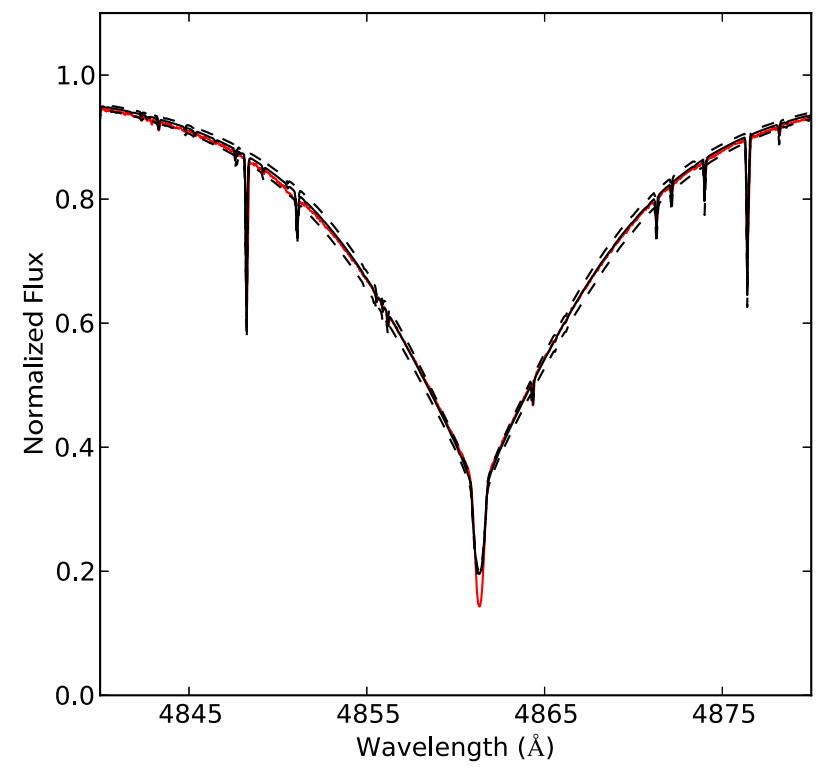

Figure 1. The fit of the $\mathrm{H} \beta$ line in the spectrum of $21 \mathrm{Peg}$. The solid red line is the observed spectrum. The dashed black is the synthetic spectrum calculated using $T_{\text {eff }}=10400 \mathrm{~K}$ and $\log g=3.55$. The two dashed blue lines are $\pm 200 \mathrm{~K}$.

removed any lines that show NLTE effects and/or have evidence of a hyperfine structure.

The results of our test are shown in Fig. 1 (which shows a comparison between our newly calculated $\mathrm{H} \beta$ profile and the observed spectrum) and in Table 5. The numerical results of Table 5 demonstrate that our results agree within the errors of those calculated by Fossati et al. (2009).

\section{OBSERVATIONS OF NGC 6250}

\subsection{Target}

The cluster NGC 6250 is located in the constellation Ara in the Southern hemisphere. Using proper motions and photometry from PPMXL (Roeser, Demleitner \& Schilbach 2010) and 2MASS (Skrutskie et al. 2006) $J H K$ photometry data, Kharchenko et al. (2013) have estimated the age of the cluster to be $\log t \sim 7.42 \mathrm{yr}$ and the distance from the Sun to the cluster as $865 \mathrm{pc}$. Kharchenko et al. (2013) calculated the cluster proper motion as $0.7 \pm 0.4 \mathrm{mas} \mathrm{yr}^{-1}$ in right ascension (RA) and $-4.1 \pm 0.4 \mathrm{mas} \mathrm{yr}^{-1}$ in declination (DEC) with a cluster radial velocity of $-8.0 \pm 0.8 \mathrm{~km} \mathrm{~s}^{-1}$. Previously, Herbst (1977) estimated $\log t \sim 7.146 \mathrm{yr}$ for the age and $1025 \mathrm{pc}$ for the distance. Moffat $\&$ Vogt (1975) estimated $d=950$ pc. NGC 6250 
Table 5. A comparison between the chemical abundances of 21 Peg calculated by Fossati et al. (2009) and those calculated by SPARTI_SIMPLE, along with the solar abundances from Asplund et al. (2009).

\begin{tabular}{|c|c|c|c|c|}
\hline \multirow[b]{2}{*}{ Element } & \multicolumn{3}{|c|}{$\log (N / \mathrm{H})$} & \multirow[b]{2}{*}{ Solar } \\
\hline & $\begin{array}{l}\text { Fossat1 et al. } \\
\quad(2009)\end{array}$ & SPARTI_SIMPLE & $\Delta$ & \\
\hline $\mathrm{O}_{\mathrm{I}}$ & $8.76 \pm 0.11$ & $8.80 \pm 0.06$ & -0.04 & 8.69 \\
\hline $\mathrm{Al}$ II & $6.34 \pm 0.10$ & $6.37 \pm 0.16$ & -0.03 & 6.45 \\
\hline Si II & $7.55 \pm 0.13$ & $7.51 \pm 0.07$ & 0.04 & 7.51 \\
\hline $\mathrm{S}_{\text {II }}$ & $7.18 \pm 0.13$ & $7.24 \pm 0.34$ & -0.06 & 7.12 \\
\hline $\mathrm{Sc}_{\text {II }}$ & $2.67 \pm 0.10$ & $2.59 \pm 0.23$ & 0.08 & 3.15 \\
\hline Ti II & $4.81 \pm 0.09$ & $4.78 \pm 0.03$ & 0.03 & 4.95 \\
\hline $\mathrm{V}_{\text {II }}$ & $4.06 \pm 0.06$ & $3.96 \pm 0.05$ & 0.10 & 3.93 \\
\hline $\mathrm{Cr}$ II & $5.84 \pm 0.10$ & $5.79 \pm 0.02$ & 0.05 & 5.64 \\
\hline $\mathrm{Fe}_{\text {II }}$ & $7.54 \pm 0.12$ & $7.52 \pm 0.01$ & 0.02 & 7.50 \\
\hline $\mathrm{Ni}$ II & $6.43 \pm 0.09$ & $6.38 \pm 0.03$ & 0.05 & 6.22 \\
\hline Sr II & $2.94 \pm-$ & $2.91 \pm 0.04$ & 0.03 & 2.87 \\
\hline Ba II & $2.85 \pm 0.06$ & $2.81 \pm 0.24$ & 0.04 & 2.18 \\
\hline
\end{tabular}

is located in a dust-rich region of space, with $E(B-V)=0.385$ and $E(J-H)=0.123$ (Kharchenko et al. 2013). To our best knowledge, the cluster has not previously been studied in detail spectroscopically, except for the purpose of classification spectra and radial velocity measurements.

\subsection{Instrument}

The observations of NGC 6250 were obtained in service mode on 2007 May 27 and 30 using FLAMES, the multi-object spectrograph attached to the Unit 2 Kueyen of the ESO/VLT.

The FLAMES instrument (Pasquini et al. 2002) is able to access targets over a field of view of 25 arcmin in diameter. Its 138 fibres feed two spectrographs, GIRAFFE and the Ultraviolet and Visual Echelle Spectrograph (UVES). This makes it possible to observe up to 138 stars, 130 using GIRAFFE linked to FLAMES with MEDUSA fibres, and 8 using UVES linked to FLAMES with UVES fibres. FLAMES-GIRAFFE can obtain low- or medium-resolution spectra $(R=7500-30000)$, within the spectral range 3700-9000. Low-resolution spectra may be obtained within wavelength intervals 500-1200 $\AA$ wide; medium-resolution spectra are obtained in wavelength intervals 170-500 ̊ wide. FLAMES-UVES can obtain high-resolution spectra $(R=47000)$, with central wavelengths of 5200,5800 or $8600 \AA$ each covering a wavelength range $\sim 2000 \AA$ wide.

\subsection{Instrument settings}

For the observations, we chose the instrument set-up that allowed us (1) to observe two hydrogen lines, which are essential to the determination of $T_{\text {eff }}$ and $\log g$ and (2) to maximize the number of metal lines and consequently the number of chemical elements available for spectral analysis. The GIRAFFE settings were chosen such that they are as close to the guiding wavelength of $520 \mathrm{~nm}$ as possible, in an effort to minimize light losses due to atmosphere differential refraction. The higher spectral resolution of UVES means that, to achieve a high enough $\mathrm{S} / \mathrm{N}$ ratio for each spectra, UVES requires an exposure time typically three or four times that of GIRAFFE. As a result, during UVES observations we are able to obtain observations with three GIRAFFE settings. The settings we used are shown in Table 6. We observed with the HR9B and HR11 settings both observing nights and one L- setting each night.
Table 6. Instrument settings information with the useful spectral lines given for stellar $T_{\text {eff }}$ between $\sim 6000 \AA$ and $\sim 25000 \AA$.

\begin{tabular}{|c|c|c|c|}
\hline $\begin{array}{l}\text { Instrument } \\
\text { setting }\end{array}$ & $\begin{array}{l}\text { Resolving } \\
\text { power }\end{array}$ & $\begin{array}{c}\text { Spectral } \\
\text { region }(\AA)\end{array}$ & $\begin{array}{l}\text { Important } \\
\text { spectral lines }\end{array}$ \\
\hline LR3 & 7500 & $4500-5077$ & $\mathrm{H} \beta$ \\
\hline HR9B & 25900 & $5139-5355$ & $\begin{array}{l}\text { Fe-peak (inc. Fe, Ti and Cr) } \\
\text { Mg triplet at } \lambda \text { 's } \\
\sim 5167,5172 \text { and } 5183 \AA\end{array}$ \\
\hline HR11 & 24200 & $5592-5838$ & $\mathrm{Fe}, \mathrm{Na}$ and $\mathrm{Sc}$ \\
\hline LR6 & 8600 & $6438-6822$ & $\mathrm{H} \alpha$ \\
\hline UVES & 47000 & $4140-6210$ & $\begin{array}{l}\mathrm{H} \beta, \mathrm{H} \gamma \\
\text { Fe-peak (inc. Fe, Ti and } \mathrm{Cr} \text { ) } \\
\text { Mg triplet at } \lambda \text { 's } \\
\sim 5167,5172 \text { and } 5183 \AA\end{array}$ \\
\hline
\end{tabular}

To prevent saturation of the observations, each UVES observation was divided into four sub-exposures and each GIRAFFE setting was divided into two subexposures.

\subsection{Data reduction}

Our data were obtained in service mode, and the package released included the products reduced by ESO with the instrument dedicated pipelines. In this work, we used the low- and mid-resolution GIRAFFE data as reduced by ESO and re-reduced UVES spectra.

For the normalization, we followed a standard procedure. First, we fit the observed spectrum with a function $G(\lambda)$, clipping in an iterative way all points $3 \sigma$ above and $1 \sigma$ below the spectrum. Then, we compare the normalized spectrum with a synthetic spectrum calculated adopting a similar stellar model, in order to test the quality of our normalization. Experience has shown for the lowresolution settings LR3 and LR6, a third-order polynomial gives the best normalization. For UVES and the high-resolution settings HR9B and HR11, a cubic spline gives the best normalization. In the case of UVES, the Balmer lines spread across two different orders, the merging of the orders was therefore done before the normalization.

\section{CLUSTER MEMBERSHIP}

Previous studies by Bayer et al. (2000), Dias et al. (2006) and Feinstein et al. (2008) give cluster membership information for the stars in the vicinity of NGC 6250. Dias et al. (2006) estimate cluster membership using the statistical method of Sanders (1971). The probabilities are shown in Table 7. Note that some of the results of Dias et al. (2006) show inconsistencies: three stars (UCAC 12065057, UCAC 12284608 and UCAC 12284626) have been assigned 100 per cent likelihood of being members despite their proper motion values being far from the cluster mean (which they calculate to be consistent with Kharchenko et al. 2013). Furthermore, UCAC 12065064 has proper motion values very close to the cluster mean values and its photometry fits well with the theoretical isochrone, it only has a 3 per cent likelihood of being a member.

The determination of cluster membership from previous literature was used as a guess for an initial target selection. Our new spectroscopic data allow a refined membership study, which is critical for our analysis. 
Table 7. List of programme stars. The proper motion $(\mu)$ in right ascension (RA) and declination (DEC), for each star, is taken from the UCAC2 catalogue (Zacharias et al. 2005). The radial velocities $\left(v_{\mathrm{r}}\right)$ are calculated using the method in Section 6.1 and for the member stars using SPARTI_SIMPLE. $\Delta$ is the number of standard deviations the proper motions and radial velocity are away from the cluster mean. The $\mathrm{S} / \mathrm{N}$ (column 8 ) corresponds to the S/N ratio per spectral bin of either the GIRAFFE settings or the UVES $520 \mathrm{~nm}$ setting. The $B V$ photometry is taken from the APASS catalogue (Henden et al. 2016) and the $J K$ photometry is taken from the UCAC2 catalogue (Zacharias et al. 2005). The Memb. column gives the results of our membership analysis, where ' $y$ ' means the star is a member, ' $n$ ' means the star is not a member. The final column gives the membership probabilities given by Dias et al. (2006).

\begin{tabular}{|c|c|c|c|c|c|c|c|c|c|c|c|c|c|}
\hline \multirow{2}{*}{$\begin{array}{l}\text { Star } \\
\text { name }\end{array}$} & \multirow{2}{*}{$\begin{array}{c}\mu_{\mathrm{RA}} \\
\left({\left.\operatorname{mas~} \mathrm{yr}^{-1}\right)}^{-1}\right.\end{array}$} & \multirow[t]{2}{*}{$\Delta_{\mu_{\mathrm{RA}}}$} & \multirow{2}{*}{$\begin{array}{c}\mu_{\mathrm{DEC}} \\
\left(\operatorname{mas~yr}^{-1}\right)\end{array}$} & \multirow[t]{2}{*}{$\Delta_{\mu_{\mathrm{DEC}}}$} & \multirow{2}{*}{$\begin{array}{c}v_{\mathrm{r}} \\
\left(\mathrm{km} \mathrm{s}^{-1}\right)\end{array}$} & \multirow[t]{2}{*}{$\Delta_{v_{\mathrm{r}}}$} & \multirow{2}{*}{$\begin{array}{c}\text { S/N } \\
\text { LR3/HR9B/HR11/LR6 }\end{array}$} & \multicolumn{4}{|c|}{ Phot. (Mag) } & \multirow[b]{2}{*}{ Memb. } & \multirow{2}{*}{$\begin{array}{l}\text { Dias } \\
\text { Memb. }\end{array}$} \\
\hline & & & & & & & & $B$ & $V$ & $J$ & $K$ & & \\
\hline CD-4511088 & $2.4 \pm 1.4$ & 0.6 & $-3.5 \pm 1.4$ & 0.4 & $-36.0 \pm 0.5$ & 2.6 & $94 / 95 / 128 / 122$ & 11.4 & 11.0 & 10.4 & 10.1 & $\mathrm{n}$ & \\
\hline HD 152743 & $0.3 \pm 1.4$ & 0.0 & $-4.6 \pm 1.4$ & 0.1 & $0.4 \pm 52.4$ & 1.0 & $116 / 252 / 146 / 194$ & 9.2 & 9.1 & 8.7 & 8.6 & $\mathrm{y}$ & \\
\hline HD 329261 & $-10.0 \pm 0.5$ & 3.3 & $-19.0 \pm 0.5$ & 4.7 & $-3.1 \pm 0.5$ & 0.7 & $59 / 62 / 84 / 128$ & 11.2 & 10.8 & 9.9 & 9.6 & $\mathrm{n}$ & \\
\hline HD 329268 & $-6.0 \pm 0.5$ & 2.0 & $-7.0 \pm 0.5$ & 0.7 & $-20 \pm 3.0$ & 1.0 & $62 / 69 / 92 / 72$ & 12.0 & 11.4 & 10.6 & 10.3 & $\mathrm{n}$ & \\
\hline HD 329269 & $4.0 \pm 0.5$ & 1.1 & $-13.0 \pm 0.5$ & 2.7 & $-16 \pm 3.0$ & 0.6 & $58 / 60 / 84 / 118$ & 11.7 & 11.2 & 9.9 & 9.6 & $\mathrm{n}$ & \\
\hline NGC 6250-13 & $2.7 \pm 2.5$ & 0.7 & $-3.1 \pm 2.4$ & 0.6 & $-14 \pm 3.0$ & 0.4 & 49/59/70/58 & 13.5 & 13.1 & 12.0 & 11.6 & $\mathrm{y}$ & \\
\hline TYC 8327-565-1 & $-0.5 \pm 1.7$ & 0.3 & $-1.6 \pm 1.7$ & 1.1 & $-9.4 \pm 0.2$ & 0.1 & 65[UVES] & 11.0 & 10.8 & 10.5 & 10.4 & y & \\
\hline UCAC 12065030 & $-2.4 \pm 5.2$ & 0.9 & $-17.9 \pm 5.2$ & 4.4 & $-20 \pm 3.0$ & 1.0 & $43 / 53 / 62 / 49$ & 14.2 & 13.5 & 11.8 & 11.4 & $\mathrm{n}$ & 47 \\
\hline UCAC 12065057 & $-14.4 \pm 2.6$ & 4.6 & $-21.2 \pm 2.4$ & 5.5 & $-49 \pm 3.0$ & 3.9 & $50 / 59 / 72 / 61$ & 13.6 & 12.9 & 11.4 & 10.9 & $\mathrm{n}$ & 100 \\
\hline UCAC 12065058 & $11.9 \pm 5.2$ & 3.6 & $-14.7 \pm 5.2$ & 3.3 & $85.0 \pm 0.2$ & 9.5 & $40 / 43 / 53 / 48$ & 14.9 & 14.1 & 12.6 & 12.1 & $y^{*}$ & 79 \\
\hline UCAC 12065064 & $1.3 \pm 2.6$ & 0.3 & $-4.2 \pm 2.4$ & 0.2 & $-14.5 \pm 0.6$ & 0.5 & $45 / 54 / 62 / 54$ & 14.0 & 13.5 & 12.4 & 12.1 & $\mathrm{y}$ & 3 \\
\hline UCAC 12284546 & $1.9 \pm 5.7$ & 0.5 & $-3.4 \pm 1.8$ & 0.5 & $-16.1 \pm 2.3$ & 0.6 & $52 / 63 / 75 / 55$ & 13.3 & 12.9 & 11.9 & 11.6 & $\mathrm{y}$ & 4 \\
\hline UCAC 12284585 & $-6.2 \pm 1.4$ & 2.1 & $-8.9 \pm 1.4$ & 1.4 & $51 \pm 3.0$ & 6.1 & $48 / 54 / 65 / 62$ & 13.2 & 12.5 & 11.1 & 10.6 & $\mathrm{n}$ & 20 \\
\hline UCAC 12284589 & $-5.1 \pm 3.0$ & 1.7 & $-5.5 \pm 1.8$ & 0.2 & $-12 \pm 3.0$ & 0.2 & 76/101/104/103 & 12.1 & 11.8 & 11.2 & 11.0 & $\mathrm{y}$ & 7 \\
\hline UCAC 12284594 & $0.8 \pm 5.2$ & 0.1 & $-3.1 \pm 5.2$ & 0.6 & $9.7 \pm 0.3$ & 2.0 & $40 / 42 / 56 / 48$ & 14.6 & 13.9 & 12.5 & 12.1 & $\mathrm{y}$ & 7 \\
\hline UCAC 12284608 & $3.5 \pm 5.2$ & 1.0 & $-31.0 \pm 5.2$ & 8.7 & $-55 \pm 3.0$ & 4.5 & $36 / 38 / 44 / 49$ & 15.3 & 14.3 & 12.3 & 11.6 & $\mathrm{n}$ & 100 \\
\hline UCAC 12284620 & $-6.3 \pm 5.2$ & 2.1 & $-27.1 \pm 5.2$ & 7.4 & $-55 \pm 3.0$ & 4.5 & $41 / 46 / 56 / 55$ & 14.3 & 13.4 & 11.8 & 11.3 & $\mathrm{n}$ & 98 \\
\hline UCAC 12284626 & $-11.5 \pm 5.2$ & 3.7 & $-28.6 \pm 5.2$ & 7.9 & $-15 \pm 3.0$ & 0.5 & $40 / 45 / 49 / 56$ & 14.3 & 13.5 & 12.1 & 11.7 & $\mathrm{n}$ & 100 \\
\hline UCAC 12284628 & $2.9 \pm 1.4$ & 0.8 & $-10.5 \pm 3.4$ & 1.9 & $-45.0 \pm 0.5$ & 3.5 & $53 / 72 / 80 / 66$ & 13.0 & 12.7 & 11.8 & 11.6 & $\mathrm{y}^{*}$ & 14 \\
\hline UCAC 12284631 & $2.9 \pm 1.4$ & 0.8 & $-5.1 \pm 1.4$ & 0.1 & $-0.9 \pm 0.2$ & 0.9 & $62 / 81 / 97 / 79$ & 11.8 & 11.3 & 11.3 & 11.0 & $\mathrm{y}$ & 4 \\
\hline UCAC 12284638 & $1.0 \pm 1.5$ & 0.2 & $-1.4 \pm 1.5$ & 1.1 & $-6 \pm 3.0$ & 0.4 & $57 / 72 / 89 / 69$ & 12.0 & 11.8 & 11.7 & 11.4 & y & 3 \\
\hline UCAC 12284645 & $0.4 \pm 1.4$ & 0.0 & $-2.3 \pm 1.4$ & 0.8 & $-19.0 \pm 9.2$ & 0.9 & 65/76/96/72 & 12.8 & 12.4 & 11.5 & 11.2 & y & 2 \\
\hline UCAC 12284653 & $3.3 \pm 5.4$ & 0.9 & $-3.3 \pm 5.2$ & 0.5 & $-13.4 \pm 0.6$ & 0.3 & $36 / 36 / 38 / 42$ & & & 13.1 & 12.6 & y & 8 \\
\hline
\end{tabular}

Note. ${ }^{*}$ Star considered as members based only on photometry.

\subsection{Proper motion and radial velocity membership}

The proper motions and radial velocities of the observed stars are shown in Table 7 and are plotted in Fig. 2. In our membership analysis, we have followed two methods.

First, we have identified those stars that are within $1 \sigma$ of the mean proper motion and radial velocity values for our sample. This way we have identified 19 stars to be members of the cluster. We calculate

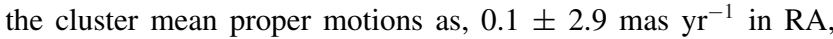
$-6.1 \pm 4.4 \mathrm{mas} \mathrm{yr}^{-1}$ in DEC and radial velocity of $-10 \pm 11 \mathrm{~km} \mathrm{~s}^{-1}$.

As a cross-check, we also use the partitional clustering technique, $K$-means clustering (MacQueen 1967). It is a technique to find common data points based on the analysis of the variables that define the data. In general, the method is to set a predicted number of data clusters and give an initial guess for the centres of these clusters. The algorithm then assigns points to the closest cluster centre and recalculates until the cluster centre values do not change. Our problem is simplified by the fact that we can define one cluster centre close to the literature value of cluster proper motion and radial velocity. In practice, we have set the number of clusters to 5: one initially centred in the literature values $\left(\mu_{0}, v_{r_{0}}\right)$ and the other four initially centred, respectively, at $\left(\mu_{0}+1.5 \sigma_{\mu}, v_{r_{0}}\right),\left(\mu_{0}-\right.$ $\left.1.5 \sigma_{\mu}, v_{r_{0}}\right),\left(\mu_{0}, v_{r_{0}}+1.5 \sigma_{v_{r}}\right)$ and $\left(\mu_{0}, v_{r_{0}}+1.5 \sigma_{v_{r}}\right)$, where $\sigma_{v_{r}}$ and $\sigma_{\mu}$ are the standard deviation in our sample. We have performed this computation using the CLUSTER function in IDL.

Fig. 3 shows the results of our cluster analysis. We found 15 members and we calculated the cluster mean proper motions as $0.4 \pm 3.0 \mathrm{mas} \mathrm{yr}^{-1}$ in RA, $-4.80 \pm 3.2 \mathrm{mas} \mathrm{yr}^{-1}$ in DEC and radial velocity of $-10 \pm 6 \mathrm{~km} \mathrm{~s}^{-1}$. Both methods give the same results apart from four stars. The discrepancy between the two methods is likely because the spread of radial velocity is large and asymmetric, and the $K$-means clustering is able to deal with this more effectively.

\subsection{Photometry}

The magnitude and colour of our sample of stars are a good indicator of cluster membership. Using the $B$ and $V$ magnitudes from Henden et al. (2016) and the $J$ and $K$ magnitudes from Zacharias et al. (2005), we have produced two colour magnitude diagrams, displayed in Fig. 4. Each diagram shows the photometry and a theoretical isochrone calculated with CMD 2.7 (Bressan et al. 2012; Chen et al. 2014, 2015; Tang et al. 2014) for an age of $\log t=7.42$. The photometry has been corrected for the 

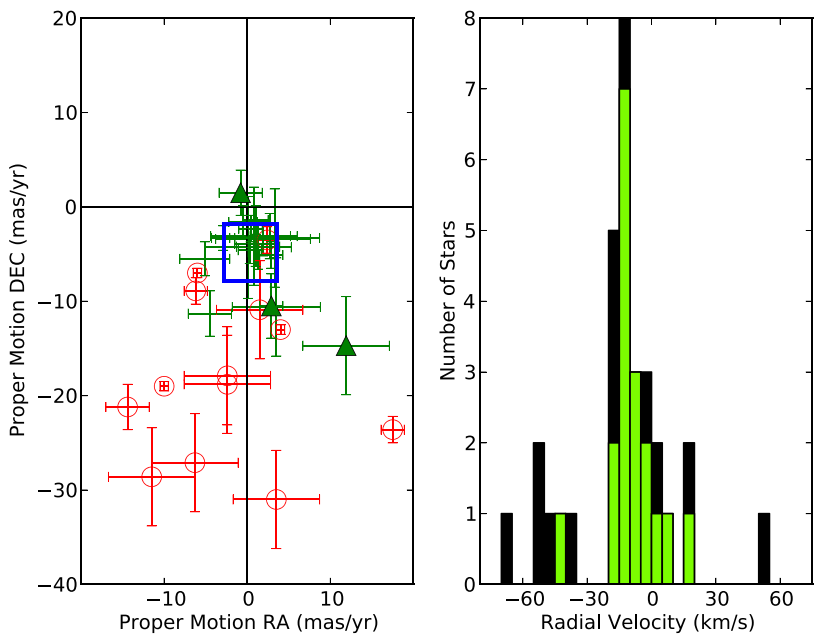

Figure 2. Left-hand panel: the proper motion of the observed stars from the UCAC2 catalogue (Zacharias et al. 2005). Green plus-signs are stars we consider to be members and red circles are stars we do not consider to be members. Triangles are the stars for which membership is considered based solely on photometry. The remaining points are stars for which membership is considered based on the kinematics and photometry. The blue box is centred at the cluster mean proper motions and represents the uncertainty of these values in each direction. Right-hand panel: histogram of the radial velocity measurements calculated using the method described in Section 6.1 for non-member stars and for the member stars using SPARTI_SIMPLE. The green histogram highlights the radial velocity of the cluster members.

extinction (Kharchenko et al. 2013) and distance to the cluster (850 pc; Kharchenko et al. 2013).

In general, we see very good agreement between the member stars determined using the kinematics approach and those
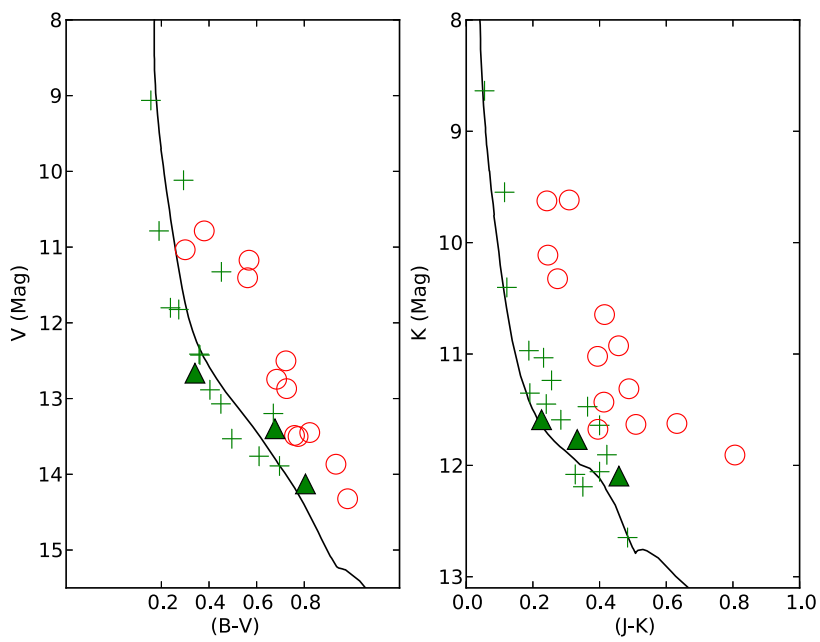

Figure 4. In both panels, green plus-signs are stars we consider members and red circles are stars we do not consider to be members. Triangles are the stars for which membership is considered based solely on photometry. The remaining points are stars for which membership is considered based on the kinematics and photometry. Left-hand panel: optical colourmagnitude diagram of the observed stars. The $B V$ photometry is taken from the APASS catalogue (Henden et al. 2016), plotted with the isochrone at ( $\log t \sim 7.42)$ corrected for $E(B-V)=0.385$ (Kharchenko et al. 2013, black solid line). Right-hand panel: infrared colour-magnitude diagram of the observed stars. The $J K$ photometry is taken from the UCAC2 catalogue (Zacharias et al. 2005), plotted with the isochrone at (log $t \sim 7.42)$ corrected for $E(J-K)=0.200$ (Kharchenko et al. 2013, black solid line).

that fit the isochrones. Notable exceptions are UCAC 12065058, UCAC 12065075 and UCAC 12284628, which do not agree with the kinematics of the cluster mean, but agree very well with the photometry. This maybe as a result of a re-ejection event and so we consider these stars as members.
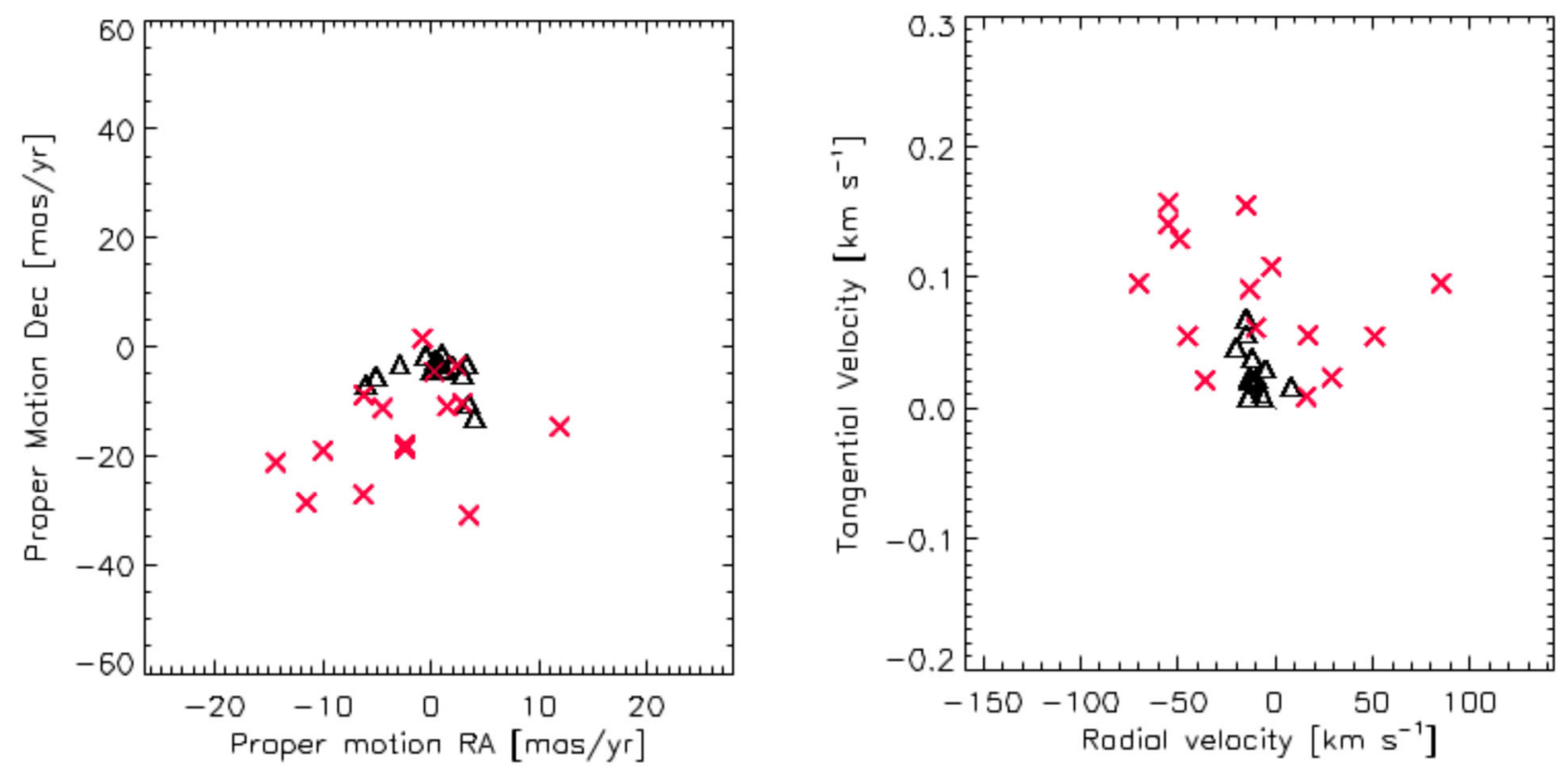

Figure 3. $K$-means clustering result for the membership analysis of NGC 6250: the black triangles are member stars and the red crosses are non-members. The left-hand panel shows the sample distribution in proper motion. The right-hand panel shows the sample distribution in velocity. The tangential velocity was computed using the distance 850 pc given by Kharchenko et al. (2013). 


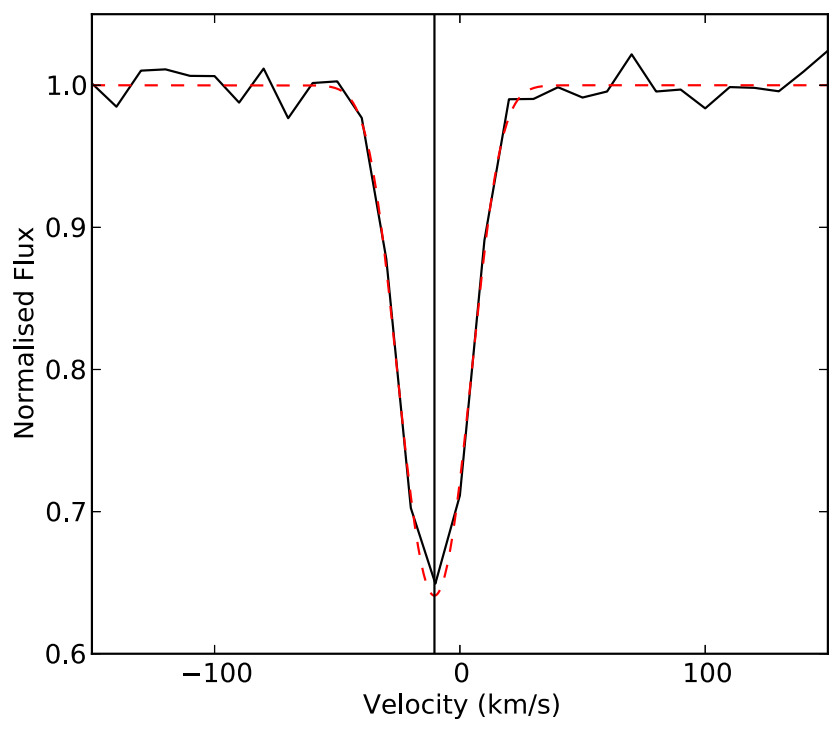

Figure 5. The least-squares deconvolution of the spectrum of UCAC $12284746\left(T_{\text {eff }}=7200 \mathrm{~K}\right)$ (solid black line), plotted against the Gaussian fit (red dashed line). The radial velocity is given by the position of the centre of the Gaussian, and it is indicated by a solid vertical line.

\section{FUNDAMENTAL PARAMETERS}

\subsection{Radial velocity and rotational velocity}

We have calculated initial values of $v_{\text {rad }}$ and $v \sin i$ from the FLAMES spectra obtained in the range 5150-5350 $\AA$ with the highest resolution setting HR9B. We used a least-squares deconvolution (LSD; Kochukhov, Makaganiuk \& Piskunov 2010) of the observed spectrum with lines selected from the VALD list (Piskunov et al. 1995) to calculate an average line profile in velocity space. To do the line selection, we must have an estimate of the temperature, so we use a combination of Balmer lines and photometry to determine an estimate of the temperature. The radial velocity of each star has been estimated using a Gaussian fit, as shown in Fig. 5.

In order to measure $v \sin i$, the LSD profile is then shifted to the rest frame, and the fast Fourier transform (FFT) is calculated. An example of the FFT is shown in Fig. 6. Following Gray (2005), the first minimum of the FFT corresponds to the stellar $v \sin i$ value. Glazunova et al. (2008) showed that it is possible to use the LSD profile, in place of the more noisy profiles of single lines, to derive the $v \sin i$ value using the FFT method described by Gray (2005). Because we expect low $v_{\text {mac }}$ values (Grassitelli et al. 2015), at the resolution of FLAMES $v_{\text {mac }}$ cannot be distinguished from $v \sin i$ and we therefore ignore it.

\subsection{Fundamental parameters from Balmer lines}

We deduced the fundamental parameters $T_{\text {eff }}$ and $\log g$ by fitting synthetic and observed Balmer lines $\mathrm{H} \beta$ and $\mathrm{H} \gamma$ for UVES spectra and $\mathrm{H} \alpha$ and $\mathrm{H} \beta$ for GIRAFFE spectra. Model atmospheres were computed with ATLAS9 (Kurucz 1993b) assuming plane parallel geometry, local thermodynamical equilibrium and opacity distribution function (ODF) for solar abundances (Kurucz 1993a). The synthetic spectra were computed with COSSAM_SIMPLE. Examples of the fit between our model and the observed Balmer lines are shown in Fig. 9.

We used hydrogen line as both temperature and gravity indicator because for $T_{\text {eff }} \lesssim 8000 \mathrm{~K}$, they are more sensitive to temperature

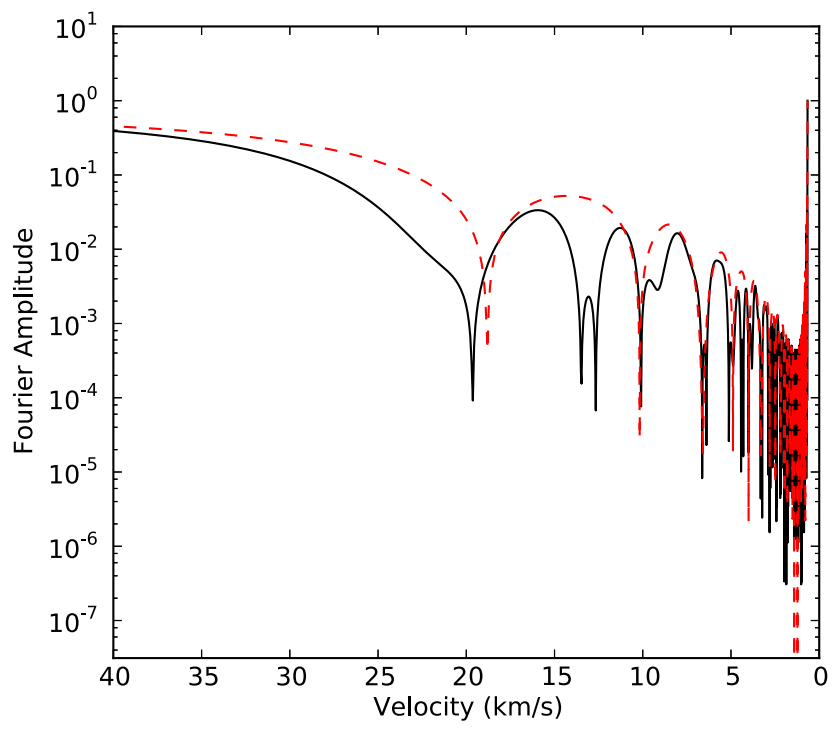

Figure 6. The fast Fourier transform of the LSD profile of Fig. 5 (black solid line), plotted with the FTT of a model LSD profile with $T_{\text {eff }}=7200 \mathrm{~K}$ and $v \sin i=19.5 \mathrm{~km} \mathrm{~s}^{-1}$ (red dashed lines).

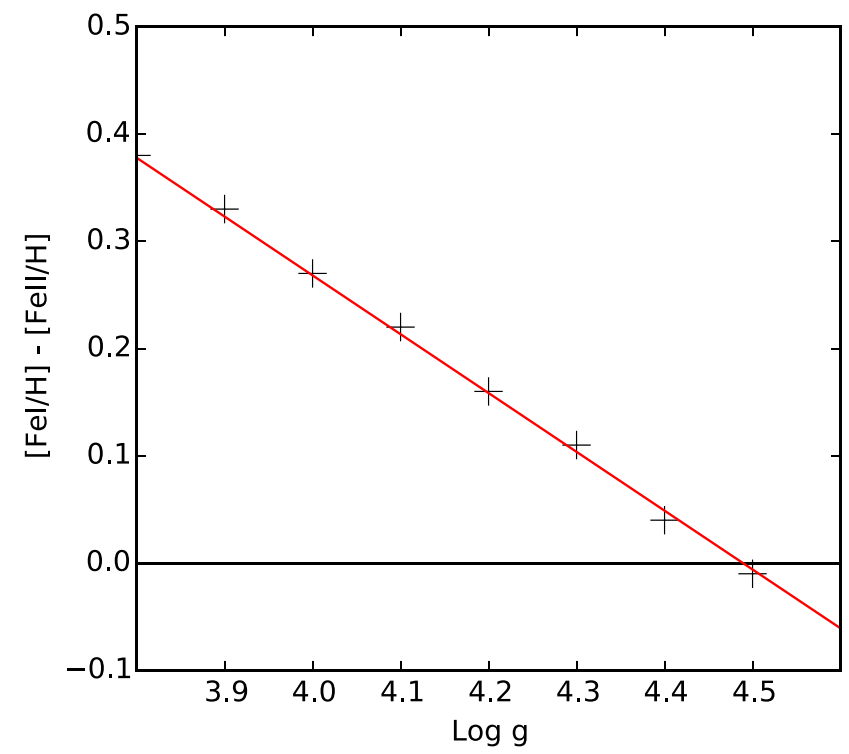

Figure 7. The difference between the abundance of Fe I and Fe II plotted as a function of surface gravity for the Sun determined using SPARTI_SIMPLE by varying the $\log g$ of the model atmosphere at $T_{\text {eff }}=5800 \mathrm{~K}$.

and for higher temperature they are more sensitive to $\log g$ variation but temperature effects can still be visible in the part of the wing close to the line core, according to Fossati et al. (2011a).

To check our values of $\log g$ for each star, we determined the $\log g$, which provided the best ionization balance between Fe I and $\mathrm{Fe}$ II lines. To test this method, we calculate the abundance of $\mathrm{Fe} I$ and $\mathrm{Fe}$ I for the Sun using $T_{\text {eff }}=5800 \mathrm{~K}$ and varying the $\log g$ between 3.8 and 4.5. The value of $\log g$ where the abundances of $\mathrm{Fe} \mathrm{I}$ and $\mathrm{Fe}$ II are equal is 4.49 compared with 4.44 found by Prsa et al. (2016). This along with our test for 21 Peg are shown in Figs 7 and 8. For each of the NGC 6250 stars, the $\log g$ values found using both methods agree within the uncertainties. 


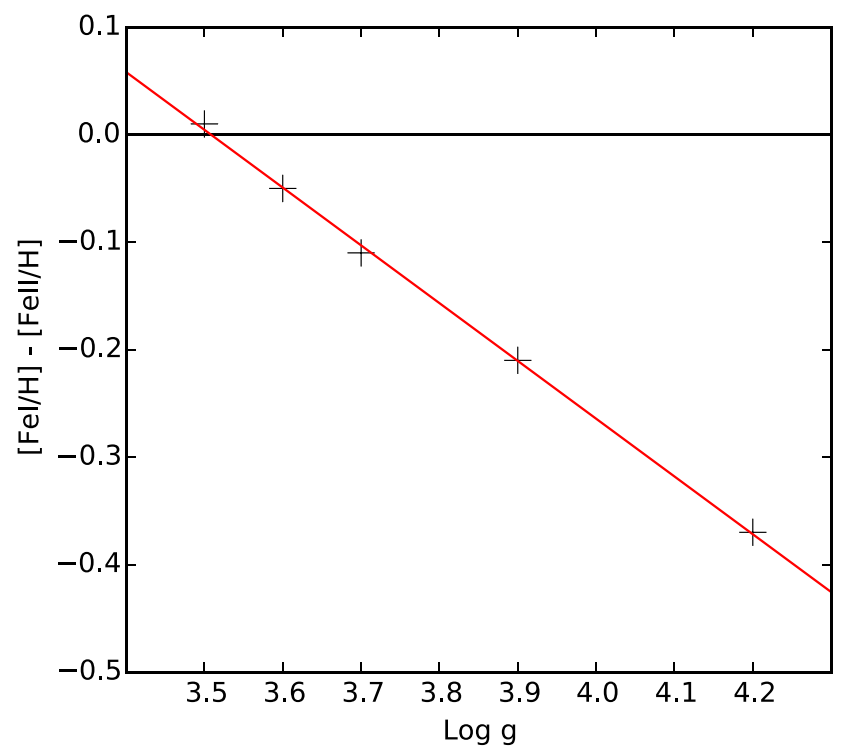

Figure 8. The difference between the abundance of $\mathrm{Fe}$ I and $\mathrm{Fe}$ II plotted as a function of surface gravity for the star 21 Peg determined using SPARTI_SIMPLE by varying the $\log g$ of the model atmosphere at $T_{\text {eff }}=10400 \mathrm{~K}$.

As a result of the low $\mathrm{S} / \mathrm{N}$, we were unable to measure any abundances for TYC8327-565-1 and UCAC 12284638; however, we were able to estimate $T_{\text {eff }}$ and $\log g$ that are given in Table 8 .

\section{RESULTS AND DISCUSSION}

The results of the abundance analysis are given in Table 9. Since this is a young cluster, there is the potential for some of the stars to still have discs. If discs were present, we would expect to see the presence of emission lines, particularly in the core of $\mathrm{H} \alpha$ and $\mathrm{H} \beta$. We do not see any evidence of emission lines in any of the stars.

\subsection{UCAC 12284546}

UCAC 12284546 shows an overabundance of C, Ca, Cr, Fe and Ni and an underabundance of $\mathrm{Mg}$. However, this abundance pattern does not match any standard chemically peculiar star in this temperature range. To better understand this star, it would be necessary to collect and analyse a higher resolution spectrum with higher $\mathrm{S} / \mathrm{N}$. As a result of the abundance anomalies we observe in this star, we do not consider this star in the global analysis of the results.

\subsection{Stellar metallicity}

For the evolutionary tracks and isochrones, we adopted the metallicity calculated as

$Z_{\text {cluster }}=10^{[\mathrm{Fe} / \mathrm{H}]_{\text {stars }}-[\mathrm{Fe} / \mathrm{H}]} \odot \mathrm{Z}_{\odot}$,

where $Z_{\text {cluster }}$ and $[\mathrm{Fe} / \mathrm{H}]_{\text {stars }}$ are, respectively, the clusters metallicity and average Fe abundance. This formulation does not follow the definition of $Z$, which is

$Z=\sum_{i=1}^{n} m_{i} X_{i}$

where $n$ is the number of elements, $m_{i}$ is the atomic mass of each element and $X_{i}$ the abundance of each element. In equation (7), $Z$ is driven mostly by the abundance of $\mathrm{C}$ and $\mathrm{O}$, which are the most

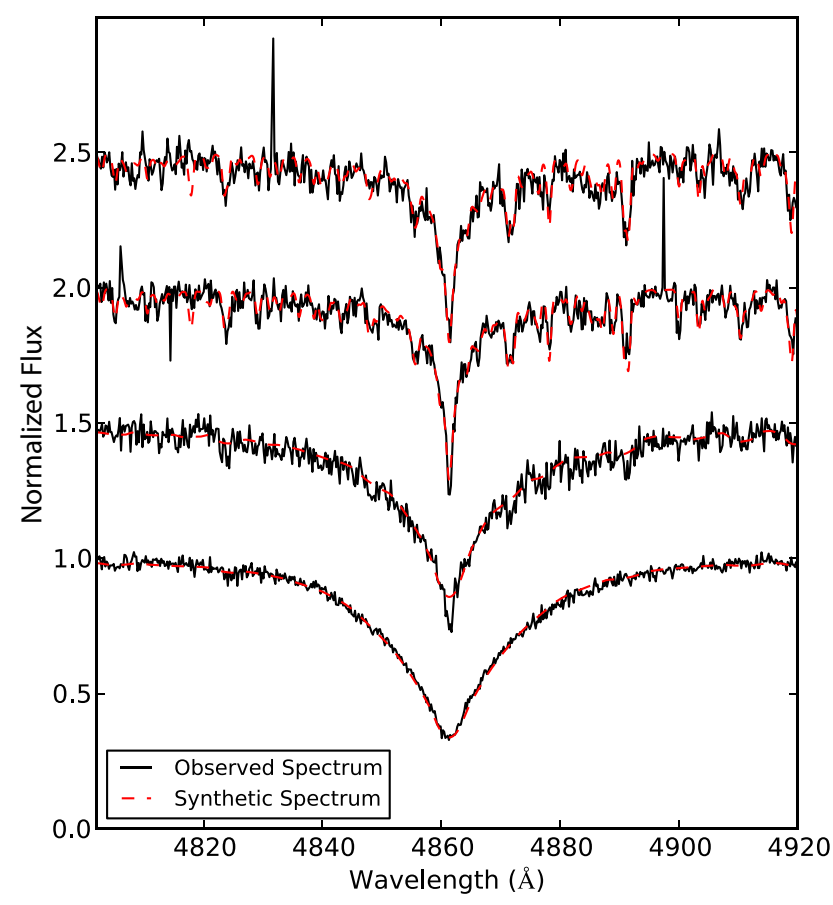

Figure 9. A sample of the observed $\mathrm{H} \beta$ lines (black solid lines) fitted with the model spectra (red dashed line). From top to bottom the stars are UCAC $12284594\left(T_{\text {eff }}=6200 \mathrm{~K}\right), \mathrm{UCAC} 12065075\left(T_{\text {eff }}=6300 \mathrm{~K}\right)$, $\mathrm{UCAC} 12065064\left(T_{\text {eff }}=7600 \mathrm{~K}\right)$ and UCAC $12284536\left(T_{\text {eff }}=9800 \mathrm{~K}\right)$. Each profile is calculated with $v \sin i$ as shown in Table 8 .

abundant elements following $\mathrm{H}$ and $\mathrm{He}$, but in stellar evolutionary calculations the relevant factor is the Fe opacity. This is why for the cluster metallicity we adopt the expression given by equation (6). When using equation (6) to infer the metallicity, it is important to use as $\mathrm{Z}_{\odot}$ the value adopted by the considered stellar evolution tracks. In this work, we use the stellar evolutionary tracks by Bressan et al. (2012), which adopt $\mathrm{Z}_{\odot}=0.0152$. Using the average $\mathrm{Fe}$ abundance obtained from the non-chemically peculiar stars, we obtain $Z_{\text {cluster }}=0.018 \pm 0.005$, which is consistent with the solar value within the uncertainty.

\subsection{Spectroscopic H-R diagram}

We plot a spectroscopic Hertzsprung-Russell (H-R) diagram (Fig. 10) using the $T_{\text {eff }}$ and $\log g$ values calculated for each star. We calculate the flux weighted luminosity, $\log \mathcal{L} / \mathcal{L}_{\odot}$, following Langer \& Kudritzki (2014) with

$\log \mathcal{L} / \mathcal{L}_{\odot}=\log \left(\frac{T_{\text {eff }}^{4}}{g}\right)-\log \left(\frac{T_{\text {eff }}^{4}}{g \odot}\right)$

where $T_{\text {eff }}$ and $\log g$ are taken from Table $8, T_{\text {eff }} \odot$ is the solar effective temperature and $g_{\odot}$ is the solar surface gravity. The isochrones are from Bressan et al. (2012). Based on the H-R diagram, we are not able to constrain the age of this cluster; however, the age of $\log t=7.42$ given by Kharchenko et al. (2013) fits well our data. As a result, we use this age in the remainder of the paper. We also give the flux-weighted luminosity, masses and fractional age of each of the stars in Table 10 calculated by fitting evolutionary tracks (Bressan et al. 2012) to each star. 
Table 8. Atmospheric parameters for the sample of stars from NGC 6250.

\begin{tabular}{lcccc}
\hline Star & $\begin{array}{c}T_{\text {eff }} \\
(\mathrm{K})\end{array}$ & $\begin{array}{c}\log g \\
(\mathrm{CGS})\end{array}$ & $\begin{array}{c}v_{\text {mic }} \\
\left(\mathrm{km} \mathrm{s}^{-1}\right)\end{array}$ & $\begin{array}{c}v \sin i \\
\left(\mathrm{~km} \mathrm{~s}^{-1}\right)\end{array}$ \\
\hline HD 152706 & $9900 \pm 200$ & $4.2 \pm 0.1$ & $1.9 \pm 0.1$ & $151.6 \pm 0.2$ \\
HD 152743 & $19800 \pm 200$ & $4.1 \pm 0.1$ & $2.1 \pm 2.5$ & $198.9 \pm 12.7$ \\
NGC 6250-13 & $8200 \pm 200$ & $4.2 \pm 0.1$ & $2.0 \pm 0.1$ & $70.1 \pm 0.8$ \\
TYC8 327-565-1 & $14200 \pm 500$ & $4.2 \pm 0.3$ & - & - \\
UCAC 12065058 & $6000 \pm 200$ & $4.4 \pm 0.1$ & $0.9 \pm 0.2$ & $4.8 \pm 0.9$ \\
UCA C12065064 & $7600 \pm 200$ & $4.2 \pm 0.1$ & $0.3 \pm 0.4$ & $54.1 \pm 0.7$ \\
UCAC 12065075 & $6300 \pm 200$ & $4.4 \pm 0.1$ & $1.0 \pm 0.1$ & $15.2 \pm 0.2$ \\
UCAC 12284506 & $6100 \pm 200$ & $4.4 \pm 0.1$ & $0.6 \pm 0.1$ & $10.6 \pm 0.2$ \\
UCAC 12284536 & $10000 \pm 200$ & $4.4 \pm 0.1$ & $2.0 \pm 0.2$ & $170.2 \pm 3.6$ \\
UCAC 12284546 & $8400 \pm 200$ & $4.2 \pm 0.1$ & $0.3 \pm 0.5$ & $146.0 \pm 1.5$ \\
UCAC 12284589 & $12600 \pm 200$ & $4.2 \pm 0.1$ & $0.5 \pm 0.7$ & $215.5 \pm 2.7$ \\
UCAC 12284594 & $6100 \pm 200$ & $4.4 \pm 0.1$ & $0.3 \pm 0.2$ & $50.3 \pm 0.5$ \\
UCAC 12284628 & $10000 \pm 200$ & $4.3 \pm 0.1$ & $0.3 \pm 0.5$ & $22.9 \pm 0.8$ \\
UCAC 12284631 & $9800 \pm 200$ & $4.2 \pm 0.1$ & $0.7 \pm 0.2$ & $17.8 \pm 0.4$ \\
UCAC 12284638 & $10800 \pm 400$ & $4.2 \pm 0.3$ & - & - \\
UCAC 12284645 & $11000 \pm 200$ & $4.3 \pm 0.1$ & $2.0 \pm 0.4$ & $270.0 \pm 3.0$ \\
UCAC 12284653 & $6200 \pm 200$ & $4.4 \pm 0.1$ & $1.5 \pm 0.2$ & $49.5 \pm 1.0$ \\
UCAC 12284662 & $7400 \pm 200$ & $4.4 \pm 0.1$ & $2.0 \pm 0.1$ & $81.4 \pm 0.9$ \\
UCAC 12284746 & $7200 \pm 200$ & $4.3 \pm 0.1$ & $1.8 \pm 0.2$ & $24.5 \pm 0.4$ \\
\hline
\end{tabular}

\subsection{Analysis of chemical abundances}

Fig. 11 shows the mean abundance of each element obtained for the F-, A- and B-type stars. The error bars are calculated as the standard deviation about the mean abundance. We consider only the measurements from Table 9 with maximum errors smaller than 0.5 .

To determine whether there is any correlation with the stellar fundamental parameters, we have compared each set of element abundances with $T_{\text {eff }}, v \sin i, M / \mathrm{M}_{\odot}$ and fractional main-sequence age. In Figs 12-14, we show abundance as a function $T_{\text {eff }}$ and in Figs 15 and 16, we show abundance as a function of $v \sin i$. After comparison between abundance and each of the fundamental parameters, we see no statistically significant patterns. This is consistent with the findings of Kilıçoğlu et al. (2016).

In addition, we have compared our results with the previous studies of the open clusters NGC 6405, NGC 5460 and Praesape performed by Kilıçoğlu et al. (2016), Fossati et al. (2011a) and Fossati et al. (2007, 2008b, 2010), respectively. This allows us to determine whether there is any evidence for correlation between cluster age and abundance. We compare our results with only these clusters since they have all been analysed within this project, and the analysis has been either fully carried out (Praesape and NGC 5460) or supervised by one of us (LF) (NGC 6405 and NGC 6250), to minimize the possibility of systematic differences between the results. To compare the results from each cluster analysis, we have offset the abundance values of the individual chemical elements according to the cluster metallicities as estimated from Fe abundances of the cluster $\mathrm{F}$ and later type stars, which should be less affected by diffusion than earlier type stars.

In NGC 6250, we found that $\mathrm{O}, \mathrm{Na}, \mathrm{Sc}, \mathrm{Ti}, \mathrm{Cr}, \mathrm{Mn}, \mathrm{Ni}, \mathrm{Zn}$ and $\mathrm{Y}$ all have solar abundances within the uncertainties, while $\mathrm{S}$ and $\mathrm{V}$ are overabundant. These results are consistent with the findings of Fossati et al. (2008b), Fossati et al. (2011a) and Kilıçoğlu et al. (2016) for the Praesepe cluster, NGC 5460 and NGC 6405, respectively (see Figs 17 and 18).

Similarly to what was found by Fossati et al. (2008b), Fossati et al. (2011a) and K1lıçoğlu et al. (2016) in the Praesepe cluster, NGC 5460 and NGC 6405, we have found an overabundance of C in the F- and A-type stars of NGC 6250. However, we do not see any trend with age (see Fig. 17).

For all of the F-type stars, we find a solar abundance of $\mathrm{Mg}$, for the A-type and B-type stars there is an underabundance of $\mathrm{Mg}$; however, there is a large spread in the results and all but two stars have approximately solar abundance, which matches with the results of the previous studies.

In agreement with previous studies, we found that in A-type stars $\mathrm{Si}$ is overabundant; however, at odds with previous studies, we found that in F-type stars $\mathrm{Si}$ is underabundant. Fig. 12 indicates the presence of a possible correlation between $T_{\text {eff }}$ and the $\mathrm{Si}$ abundance, though a further analysis reveals that this apparent correlation is not statistically significant.

For all of the F-type stars, we find a solar abundance of Ca that is consistent with the previous results. However, for the A-type stars, we find an overabundance, which is contrary to the findings of the previous studies; the origin of this is unclear.

We measure the abundance of Fe in all of our stars to be approximately solar. For both Mn and Fe, Fossati et al. (2011a) found an increase in abundance with $T_{\text {eff }}$, which we do not; this therefore may be the result of an age effect. The narrow $T_{\text {eff }}$ range of the stars analysed by Fossati et al. (2008b) for the Praesepe cluster means we are unable to provide any definite conclusions until the remaining clusters are analysed.

We measure an almost solar abundance for Ba, albeit with relatively large uncertainties. This is in contrast with the findings of Fossati et al. (2008b), Fossati et al. (2011a) and Kılıçoğlu et al. (2016) who all report overabundances. To understand each of the results together, we plot the mean abundance of Ba measured for each cluster in Fig. 18. We did not consider the stars HD 122983 and HD 123182 from NGC 5460 because of their apparent chemical peculiarities (Fossati et al. 2011a). From Fig. 18, we obtain a hint of a positive correlation of $\mathrm{Ba}$ abundance with age; however, the abundance uncertainties are too large to draw any concrete conclusion. By analysing further clusters we will be able to determine whether this effect is the result of diffusion or the different chemistry of the star-forming region for each cluster.

We measure Nd to be overabundant in four stars; however, the data from previous papers are too sparse to provide any conclusion.

Finally, we have compared our results with the study of chemically peculiar magnetic Ap stars by Bailey et al. (2014). This allows us to examine the differences and similarities between abundance trends of chemically normal and chemically peculiar stars. Bailey et al. (2014) found statistically significant trends between $\mathrm{He}$, Ti, $\mathrm{Cr}, \mathrm{Fe}, \mathrm{Pr}$ and $\mathrm{Nd}$ and stellar age. They also found a strong trend between the abundances of $\mathrm{Cr}$ and $\mathrm{Fe}$, and $T_{\text {eff. }}$ For $\mathrm{Cr}$, an underabundance was observed for stars with $T_{\text {eff }} \lesssim 7000 \mathrm{~K}$, for stars with $T_{\text {eff }} \gtrsim 7000 \mathrm{~K}$ the abundance of $\mathrm{Cr}$ sharply rises and peaks at $T_{\text {eff }} \sim 10000 \mathrm{~K}$ before falling back to approximately solar. For Fe, an underabundance was observed for stars with $T_{\text {eff }} \lesssim 8000 \mathrm{~K}$ and an overabundance for the remaining stars. These results are in stark contrast with what we observed for NGC 6250. This suggests that the abundance of chemical elements in the photosphere of chemically normal F-, A- and B-type stars remains relatively constant during their main-sequence lifetime except when influenced by a magnetic field.

\section{CONCLUSIONS}

We have presented the new code for spectral analysis, SPARTI_SIMPLE. Based on COSSAM_SIMPLE, a modified version of the radiative transfer code COSSAM, SPARTI_SIMPLE employs the inversion algorithm LMA 
Table 9. The abundance of elements for the analysed stars of NGC 6250 (ordered by decreasing $T_{\text {eff }}$ ), given in $\log (N / \mathrm{H})$ where $\mathrm{H}=12.00$. In parenthesis, the first number is the error calculated using equation (3) and the second in the error calculating using equation (5). Both errors are in units of 0.01 dex. The last row of each set gives the solar abundances from Asplund et al. (2009).

\begin{tabular}{|c|c|c|c|c|c|c|c|c|}
\hline Star & $\begin{array}{l}T_{\text {eff }} \\
(\mathrm{K})\end{array}$ & $\begin{array}{c}v \sin i \\
\left(\mathrm{~km} \mathrm{~s}^{-1}\right)\end{array}$ & $\mathrm{C}$ & $\mathrm{O}$ & $\mathrm{Na}$ & $\mathrm{Mg}$ & $\mathrm{Si}$ & S \\
\hline HD 152743 & 19800 & $198.9 \pm 12.7$ & & & & & $8.07(95 ; 102)$ & \\
\hline UCAC 12284645 & 11000 & $270.0 \pm 3.0$ & & & & & & \\
\hline UCAC 12284628 & 10000 & $22.9 \pm 0.8$ & & & & $6.41(06 ; 12)$ & & \\
\hline UCAC 12284536 & 10000 & $170.2 \pm 3.5$ & & & & $7.58(25 ; 27)$ & & \\
\hline HD 152706 & 9900 & $151.6 \pm 0.2$ & $8.91(02 ; 34)$ & $8.68(02 ; 04)$ & & $7.99(02 ; 28)$ & $7.83(02 ; 27)$ & \\
\hline UCAC 12284546 & 8400 & $146.0 \pm 1.5$ & $8.83(09 ; 15)$ & & & $6.19(15 ; 67)$ & & \\
\hline NGC 6250-13 & 8200 & $70.1 \pm 0.8$ & & & & $7.74(06 ; 14)$ & & \\
\hline UCAC 12065064 & 7600 & $54.1 \pm 0.7$ & $8.63(11 ; 15)$ & & & $7.29(08 ; 13)$ & $7.39(08 ; 15)$ & $7.92(11 ; 59)$ \\
\hline UCAC 12284662 & 7400 & $81.4 \pm 0.9$ & $8.50(19 ; 37)$ & & $6.24(17 ; 20)$ & $7.59(09 ; 13)$ & $7.51(10 ; 10)$ & \\
\hline UCAC 12284746 & 7200 & $24.5 \pm 0.4$ & $8.92(13 ; 14)$ & & $6.25(13 ; 15)$ & $7.68(10 ; 15)$ & $7.26(10 ; 11)$ & \\
\hline UCAC 12065075 & 6300 & $15.2 \pm 0.2$ & & & $6.49(07 ; 22)$ & $7.64(04 ; 22)$ & $7.34(04 ; 19)$ & \\
\hline Solar & 5777 & 1.2 & 8.43 & 8.69 & 6.24 & 7.60 & 7.51 & 7.12 \\
\hline Star & $\begin{array}{l}T_{\text {eff }} \\
(\mathrm{K})\end{array}$ & $\begin{array}{c}v \sin i \\
\left(\mathrm{~km} \mathrm{~s}^{-1}\right)\end{array}$ & $\mathrm{Ca}$ & $\mathrm{Sc}$ & $\mathrm{Ti}$ & $\mathrm{V}$ & $\mathrm{Cr}$ & $\mathrm{Mn}$ \\
\hline HD 152743 & 19800 & $198.9 \pm 12.7$ & & & & & & \\
\hline UCAC 12284589 & 12600 & $215.5 \pm 2.7$ & & & & & $6.25(14 ; 18)$ & \\
\hline UCAC 12284645 & 11000 & $270.0 \pm 3.0$ & & & $4.96(28 ; 35)$ & & $5.64(23 ; 32)$ & \\
\hline UCAC 12284628 & 10000 & 22.9 & $0.8 \pm$ & & $4.94(11 ; 16)$ & & $5.57(09 ; 10)$ & \\
\hline UCAC 12284536 & 10000 & $170.2 \pm 3.5$ & & & $5.02(23 ; 23)$ & & $5.73(21 ; 23)$ & \\
\hline HD 152706 & 9900 & 151.6 & $0.2 \pm 6.92(02 ; 46)$ & & $4.95(02 ; 04)$ & & $5.78(02 ; 16)$ & \\
\hline UCAC 12284631 & 9800 & $17.8 \pm 0.4$ & & & $4.75(06 ; 08)$ & & $5.49(07 ; 08)$ & \\
\hline UCAC 12284546 & 8400 & $146.0 \pm 1.5$ & $7.14(22 ; 22)$ & & $5.05(09 ; 19)$ & & $6.14(07 ; 20)$ & \\
\hline NGC 6250-13 & 8200 & $70.1 \pm 0.8$ & $6.22(08 ; 14)$ & & $4.83(05 ; 08)$ & & $5.75(06 ; 09)$ & $5.51(13 ; 17)$ \\
\hline Solar & 5777 & 1.2 & 6.34 & 3.15 & 4.95 & 3.93 & 5.64 & 5.43 \\
\hline Star & $\begin{array}{l}T_{\text {eff }} \\
(\mathrm{K})\end{array}$ & $\begin{array}{c}v \sin i \\
\left(\mathrm{~km} \mathrm{~s}^{-1}\right)\end{array}$ & $\mathrm{Fe}$ & $\mathrm{Ni}$ & $\mathrm{Zn}$ & $\mathrm{Y}$ & $\mathrm{Ba}$ & $\mathrm{Nd}$ \\
\hline HD 152743 & 19800 & $198.9 \pm 12.7$ & $7.67(47 ; 49)$ & & & & & \\
\hline UCAC 12284589 & 12600 & $215.5 \pm 2.7$ & $7.77(04 ; 04)$ & & & & & \\
\hline UCAC 12284645 & 11000 & $270.0 \pm 3.0$ & $7.50(12 ; 23)$ & & & & & \\
\hline UCAC 12284628 & 10000 & $22.9 \pm 0.8$ & $7.43(06 ; 10)$ & & & & & \\
\hline UCAC 12284536 & 10000 & $170.2 \pm 3.5$ & $7.56(16 ; 17)$ & & & & & \\
\hline HD 152706 & 9900 & $151.6 \pm 0.2$ & $7.61(01 ; 12)$ & & & & & \\
\hline UCAC 12284631 & 9800 & $17.8 \pm 0.4$ & $7.41(03 ; 06)$ & & & & & \\
\hline UCAC 12284546 & 8400 & $146.0 \pm 1.5$ & $7.72(07 ; 23)$ & $6.94(10 ; 17)$ & & & & \\
\hline NGC 6250-13 & 8200 & $70.1 \pm 0.8$ & $7.54(04 ; 08)$ & $6.33(08 ; 13)$ & & & $2.09(42 ; 45)$ & \\
\hline UCAC 12065064 & 7600 & $54.1 \pm 0.7$ & $7.74(05 ; 14)$ & $6.16(11 ; 13)$ & & & & \\
\hline UCAC 12284662 & 7400 & $81.4 \pm 0.9$ & $7.51(07 ; 10)$ & $6.27(10 ; 28)$ & & & $2.34(36 ; 38)$ & \\
\hline UCAC 12284746 & 7200 & $24.5 \pm 0.4$ & $7.73(07 ; 11)$ & $6.30(08 ; 12)$ & $4.71(44 ; 46)$ & $2.54(13 ; 14)$ & $2.18(49 ; 49)$ & \\
\hline UCAC 12065075 & 6300 & $15.2 \pm 0.2$ & $7.65(03 ; 19)$ & $6.28(04 ; 14)$ & $4.06(33 ; 34)$ & $2.38(11 ; 14)$ & $2.18(17 ; 17)$ & $1.76(09 ; 12)$ \\
\hline UCAC 12284653 & 6200 & $49.5 \pm 1.0$ & $7.70(09 ; 20)$ & $6.44(11 ; 27)$ & $4.62(88 ; 88)$ & & $2.20(41 ; 43)$ & \\
\hline UCAC 12284594 & 6100 & $50.3 \pm 0.5$ & $7.54(02 ; 09)$ & $6.15(06 ; 07)$ & & & $2.41(13 ; 14)$ & $1.98(14 ; 17)$ \\
\hline UCAC 12284506 & 6100 & $10.6 \pm 0.2$ & $7.28(01 ; 07)$ & $5.99(03 ; 05)$ & $4.69(28 ; 28)$ & $2.07(09 ; 11)$ & $2.42(09 ; 09)$ & $1.61(07 ; 13)$ \\
\hline UCAC 12065058 & 6000 & $4.8 \pm 0.9$ & $7.39(06 ; 09)$ & $6.02(10 ; 11)$ & & & $2.18(39 ; 41)$ & $1.63(25 ; 25)$ \\
\hline Solar & 5777 & 1.2 & 7.50 & 6.22 & 4.56 & 2.21 & 2.18 & 1.42 \\
\hline
\end{tabular}




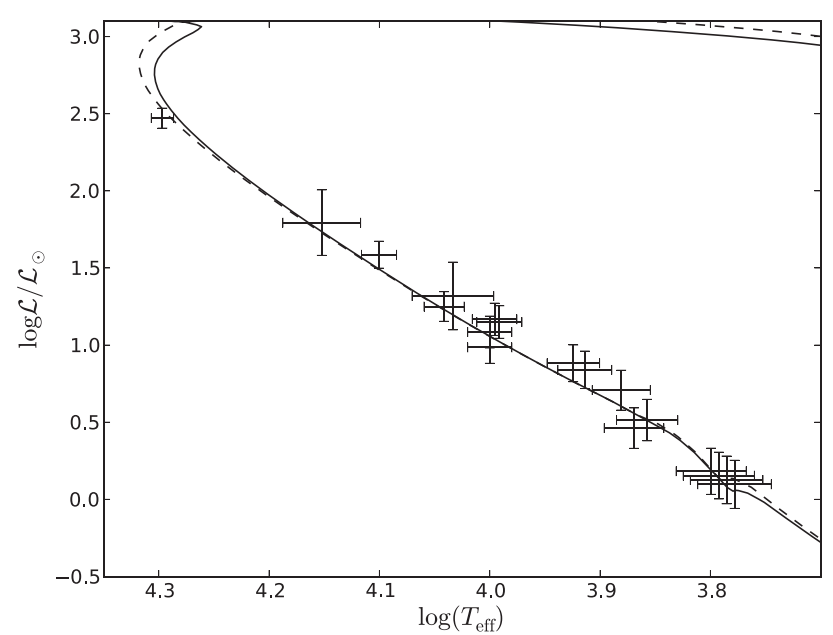

Figure 10. An H-R diagram of NGC 6250, the stars plotted (black plussigns) with theoretical isochrones (Bressan et al. 2012) at $\log t=7.40$ (dashed black line) and $\log t=7.45$ (solid black line). Both isochrones have solar metallicity.

Table 10. $\log \mathcal{L} / \mathcal{L}_{\odot}, \log T_{\text {eff }}, M / \mathrm{M}_{\odot}$ and fractional age $(\tau)$ with associated error bars for the stars of the NGC 6250 open cluster.

\begin{tabular}{lcccc}
\hline Star & $\log \mathcal{L} / \mathcal{L}_{\odot}$ & $\log T_{\text {eff }}$ & $M / \mathrm{M}_{\odot}$ & $\tau$ \\
\hline HD 152706 & $1.17 \pm 0.10$ & $4.00 \pm 0.02$ & $2.25 \pm 1.00$ & $0.02 \pm 0.04$ \\
HD 152743 & $2.47 \pm 0.06$ & $4.30 \pm 0.01$ & $6.40 \pm 0.60$ & $0.44 \pm 0.09$ \\
NGC 6250-13 & $0.84 \pm 0.12$ & $3.91 \pm 0.02$ & $1.75 \pm 1.00$ & $0.01 \pm 0.03$ \\
TYC 8327-565-1 & $1.79 \pm 0.21$ & $4.15 \pm 0.04$ & $3.80 \pm 0.20$ & $0.12 \pm 0.14$ \\
UCAC 12065058 & $0.10 \pm 0.16$ & $3.78 \pm 0.03$ & $1.05 \pm 0.10$ & $-0.02 \pm 0.01$ \\
UCAC 12065064 & $0.71 \pm 0.13$ & $3.88 \pm 0.03$ & $1.60 \pm 0.05$ & $0.00 \pm 0.02$ \\
UCAC 12065075 & $0.18 \pm 0.15$ & $3.80 \pm 0.03$ & $1.15 \pm 0.05$ & $-0.01 \pm 0.02$ \\
UCAC 12284506 & $0.13 \pm 0.15$ & $3.79 \pm 0.03$ & $1.05 \pm 0.10$ & $-0.02 \pm 0.01$ \\
UCAC 12284536 & $0.98 \pm 0.10$ & $4.00 \pm 0.02$ & $2.05 \pm 0.10$ & $0.02 \pm 0.04$ \\
UCAC 12284546 & $0.88 \pm 0.12$ & $3.92 \pm 0.02$ & $1.80 \pm 1.00$ & $0.01 \pm 0.03$ \\
UCAC 12284589 & $1.59 \pm 0.09$ & $4.10 \pm 0.02$ & $3.20 \pm 1.00$ & $0.07 \pm 0.01$ \\
UCAC 12284594 & $0.13 \pm 0.15$ & $3.79 \pm 0.03$ & $1.05 \pm 0.10$ & $-0.02 \pm 0.01$ \\
UCAC 12284628 & $1.08 \pm 0.10$ & $4.00 \pm 0.02$ & $2.20 \pm 0.10$ & $0.02 \pm 0.04$ \\
UCAC 12284631 & $1.15 \pm 0.11$ & $3.99 \pm 0.02$ & $2.20 \pm 1.00$ & $0.02 \pm 0.04$ \\
UCAC 12284638 & $1.32 \pm 0.22$ & $4.03 \pm 0.04$ & $2.60 \pm 0.20$ & $0.04 \pm 0.06$ \\
UCAC 12284645 & $1.25 \pm 0.10$ & $4.04 \pm 0.02$ & $2.40 \pm 0.20$ & $0.03 \pm 0.05$ \\
UCAC 12284653 & $0.15 \pm 0.15$ & $3.79 \pm 0.03$ & $1.10 \pm 0.05$ & $-0.02 \pm 0.01$ \\
UCAC 12284662 & $0.46 \pm 0.13$ & $3.87 \pm 0.03$ & $1.40 \pm 0.10$ & $-0.00 \pm 0.02$ \\
UCAC 12284746 & $0.51 \pm 0.13$ & $3.86 \pm 0.03$ & $1.45 \pm 0.05$ & $-0.00 \pm 0.02$ \\
\hline
\end{tabular}

and allows one to recover the abundance of the chemical elements of non-magnetic stellar atmospheres. To test our new code, we have performed the abundance analysis of the Sun, HD 32115 and 21 Peg and compared our results with those previously published in the thorough works by Prsa et al. (2016), Asplund et al. (2009), Fossati et al. (2011b) and Fossati et al. (2009), finding excellent agreement.

We have applied our new code for a spectroscopic study of the open cluster NGC 6250, which was observed with the FLAMES instrument of the ESO VLT. From the observed sample of stars, we have performed cluster membership analysis based on a $K$-means clustering procedure and analysis of the photometry. As a result of our analysis, we selected 19 stars from our sample as members of the cluster. We have computed the cluster mean proper motions

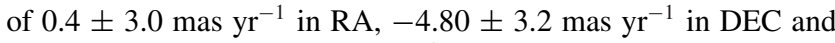
radial velocity of $-10 \pm 6 \mathrm{~km} \mathrm{~s}^{-1}$. These values agree within the errors with the values calculated by Kharchenko et al. (2013). The

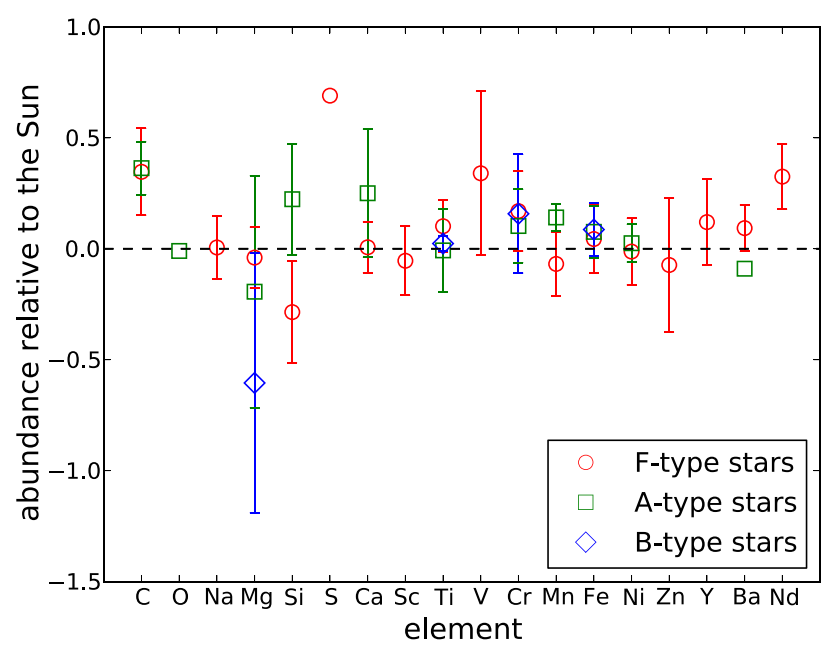

Figure 11. The mean abundances of each element relative to solar for F(red circles), A-(green squares) and B- (blue diamonds) type stars. The error bars are calculated by taking the standard deviations of the calculated mean abundances.

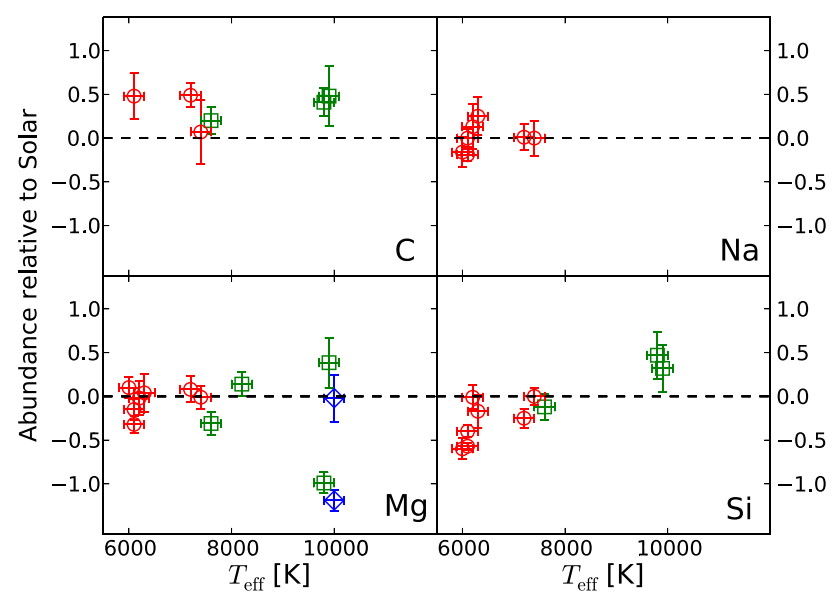

Figure 12. The abundances of $\mathrm{C}, \mathrm{Na}, \mathrm{Mg}$, and $\mathrm{Si}$ relative to the solar abundance (Asplund et al. 2009) against $T_{\text {eff. }}$ For F-(red circles), A-(green squares) and B-(blue diamonds) type stars.

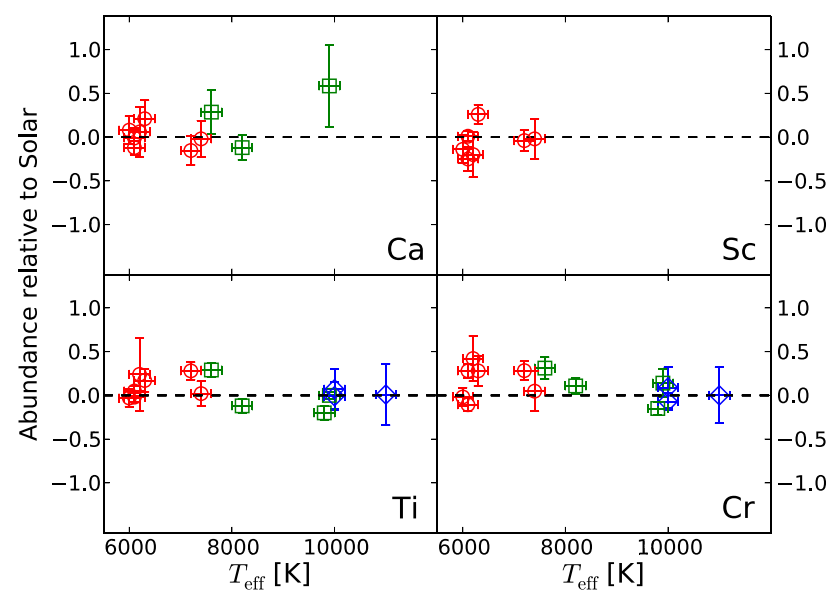

Figure 13. Same as Fig. 12, but for $\mathrm{Ca}, \mathrm{Sc}, \mathrm{Ti}$ and $\mathrm{Cr}$. 


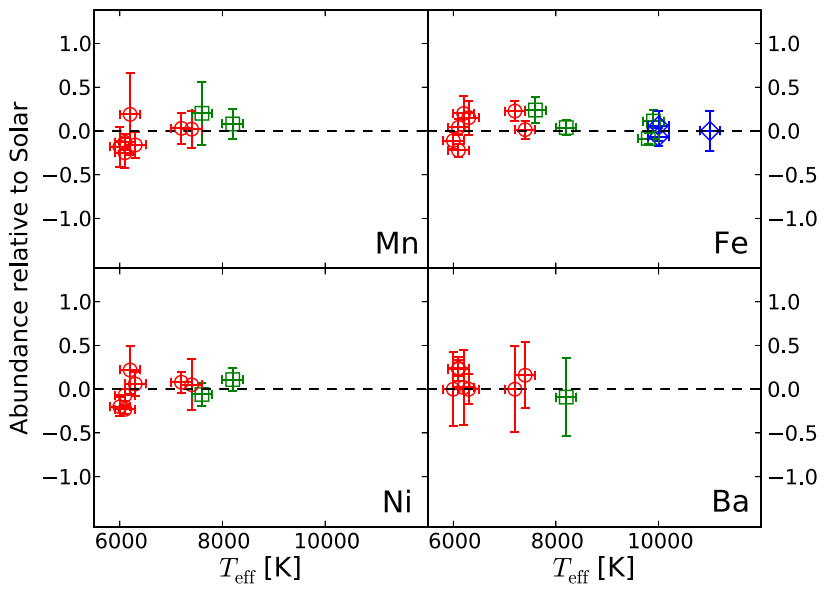

Figure 14. Same as Fig. 12, but for $\mathrm{Mn}, \mathrm{Fe}, \mathrm{Ni}$ and $\mathrm{Ba}$.

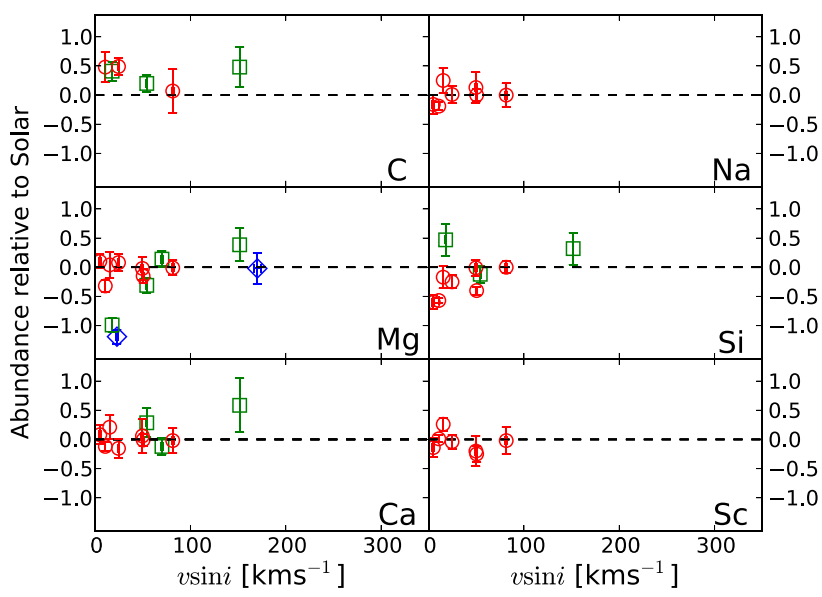

Figure 15. The abundances of $\mathrm{C}, \mathrm{Na}, \mathrm{Mg}, \mathrm{Si}, \mathrm{Ca}$ and $\mathrm{Sc}$ relative to the solar abundance (Asplund et al. 2009) against $v \sin i$. For F-(red circles), A-(green squares) and B-(blue diamonds) type stars.

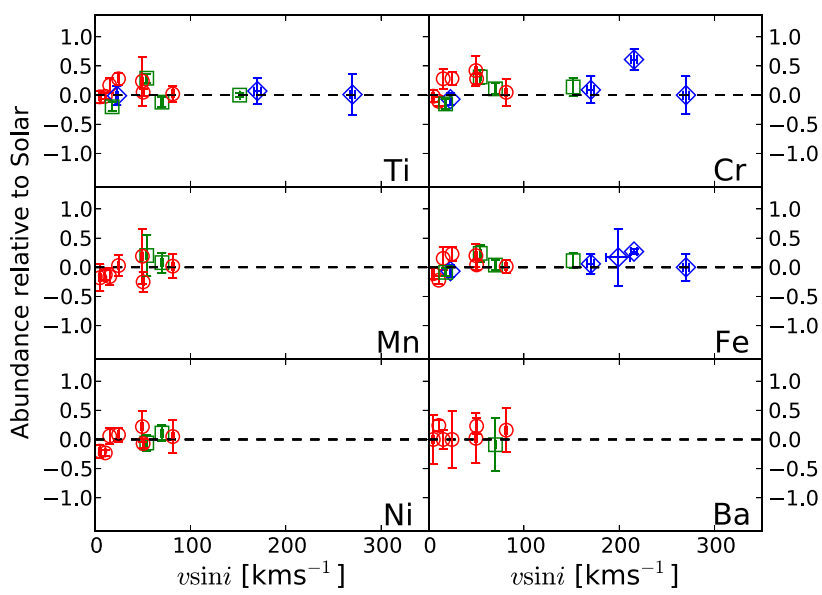

Figure 16. Same as Fig. 15, but for $\mathrm{Ti}, \mathrm{Cr}, \mathrm{Mn}, \mathrm{Fe}, \mathrm{Ni}$ and $\mathrm{Ba}$.

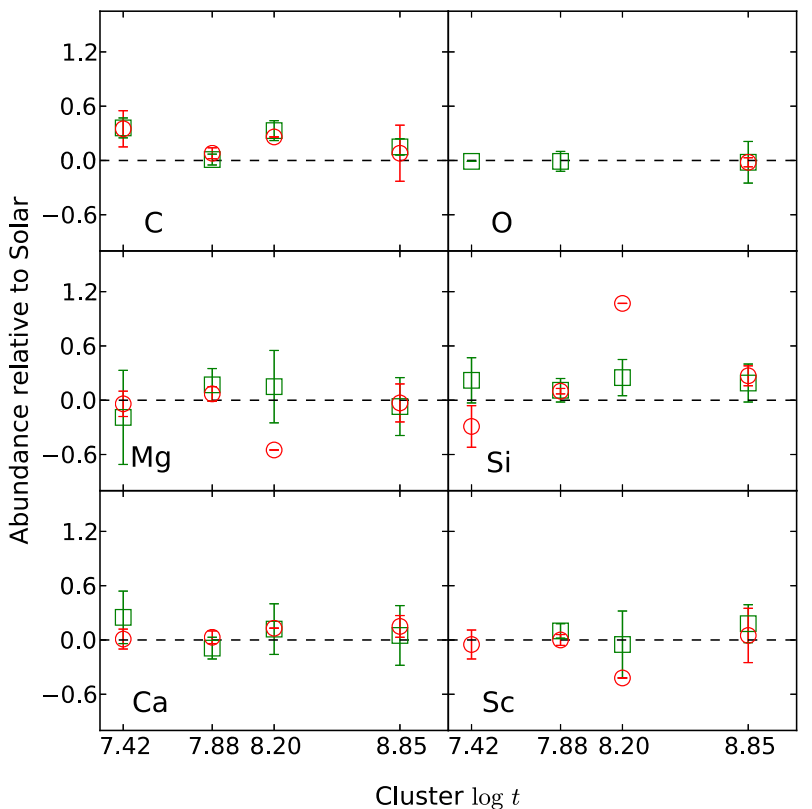

Figure 17. A comparison between the mean $\mathrm{C}, \mathrm{O}, \mathrm{Mg}, \mathrm{Si}, \mathrm{Ca}$ and $\mathrm{Sc}$ abundances found for each of the previous studies and those found in this paper. Mean abundances for F-(red circles) and A-type (green squares) stars are plotted against cluster age $(\log t=7.42$ for NGC 6250; $\log t=7.88$ for NGC $6405 ; \log t=8.20$ for NGC 5460; and $\log t=8.85$ for Praesepe). The error is given by the standard deviation of all the measured abundances.

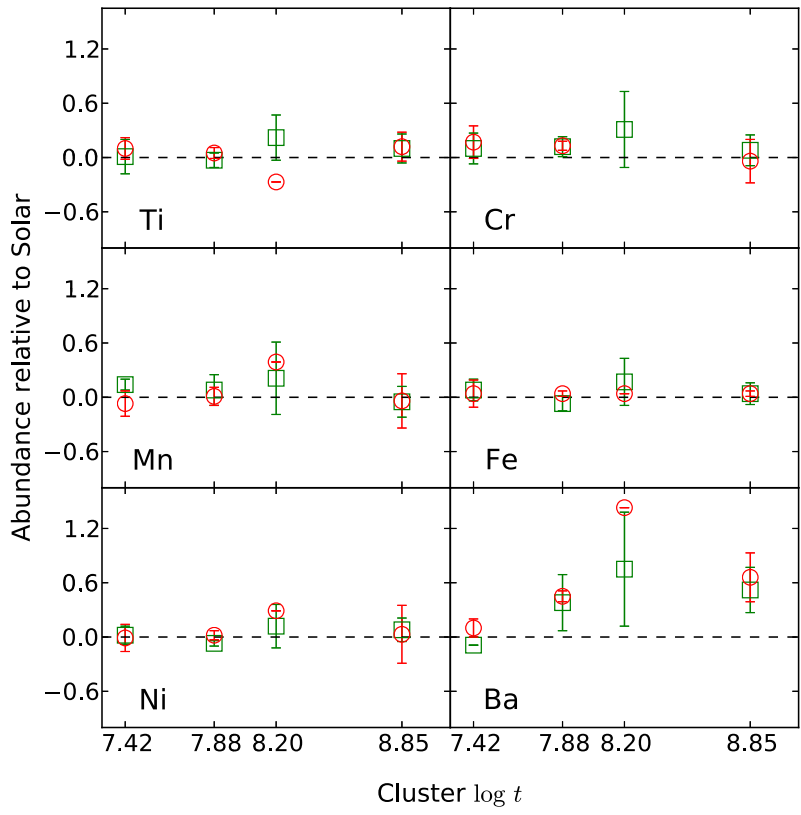

Figure 18. Same as Fig. 18 but for Ti, Cr, Mn, Fe, Ni and Ba.

age and distance given by Kharchenko et al. (2013) agree well with our photometric analysis of the cluster.

Finally, we have examined the chemical abundance measurements for each star and searched for any trend between abundance and the stellar fundamental parameters and between the abundance measured in this study and the abundance measured in the previous studies of older clusters by Fossati et al. (2007, 2008b, 2010), Fossati et al. (2011a) and Kılıçoğlu et al. (2016). Our results 
for the abundance of $\mathrm{O}, \mathrm{Na}, \mathrm{Sc}, \mathrm{Ti}, \mathrm{Cr}, \mathrm{Mn}, \mathrm{Ni}, \mathrm{Zn}$ and $\mathrm{Y}$ are solar within the uncertainties, while $\mathrm{S}$ and $\mathrm{V}$ are overabundant. These results are consistent with previous studies. We do not find evidence of the correlation between either the Fe or Mn abundance and $T_{\text {eff }}$ found by Fossati et al. (2011a); however, this may be evidence of an age effect and we need to study more clusters before being able to determine this. We find hints of an increase in mean Ba abundance with cluster age but more clusters should be analysed to confirm this trend. Comparing our results with those from Bailey et al. (2014), who searched for trends between chemical abundances and stellar parameters of chemically peculiar magnetic Ap stars, suggests that the abundance of chemical elements in the photosphere of chemically normal F-, A- and B-type stars remains relatively constant during their main-sequence lifetime except when influenced by a magnetic field.

\section{ACKNOWLEDGEMENTS}

This paper is based on observations made with ESO Telescopes at the Paranal Observatory under programme ID 079.D-0178. We thank Claudia Paladini for the re-reduction of the UVES spectra. AM acknowledges the support of a Science and Technology Facilities Council (STFC) PhD studentship. Thanks go to AdaCore for providing the GNAT GPL Edition of its Ada compiler. We thank the referee Charles Proffitt for providing constructive comments that led to a significant improvement of the manuscript. This publication makes use of data products from the AAVSO Photometric All Sky Survey (APASS). Funded by the Robert Martin Ayers Sciences Fund and the National Science Foundation.

\section{REFERENCES}

Alecian G., Stift M. J., 2004, A\&A, 416, 703

Asplund M., Grevesse N., Sauval A. J., Scott P., 2009, ARA\&A, 47, 481

Aster R. C., Borchers B., Thurber C. H., 2013, Parameter Estimation and Inverse Problems. Elsevier, New York

Auer L. H., Heasley J. N., House L. L., 1977, ApJ, 216, 531

Bagnulo S., Landstreet J. D., Mason E., Andretta V., Silaj J., Wade G. A., 2006, A\&A, 450, 777

Bailey J. D., Landstreet J. D., Bagnulo S., 2014, A\&A, 561, A147

Baschek B., Holweger H., Traving G., 1966, Astron. Abh. Hamburger Sternwarte, 8,26

Bayer C., Maitzen H., Paunzen E., Rode-Paunzen M., Sperl M., 2000, A\&AS, 147, 99

Bischof K. M., 2005, Mem. Soc. Astron. Ital. Suppl., 8, 64

Bressan A., Marigo P., Girardi L., Salasnich B., Dal Cero C., Rubele S., Nanni A., 2012, MNRAS, 427, 127

Chen Y., Girardi L., Bressan A., Marigo P., Barbieri M., Kong X., 2014, MNRAS, 444, 2525

Chen Y., Bressan A., Girardi L., Marigo P., Kong X., Lanza A., 2015, MNRAS, 452, 1068

Chmielewski Y., 1979, Spectres Stellaires Synthetiques: Programmes de Calcul Chmielewski. L'Observatoire de Geneve, Geneva

Dappen W., Anderson L., Mihalas D., 1987, ApJ, 319, 195

Dias W. S., Assafin M., Flório V., Alessi B. S., Líbero V., 2006, A\&A, 446, 949

Feinstein C., Vergne M. M., Martínez R., Orsatti A. M., 2008, MNRAS, 391,447

Fensl R. M., 1995, A\&AS, 112, 191

Folsom C. P., Wade G. A., Bagnulo S., Landstreet J. D., 2007, MNRAS, 376,361

Fossati L., Bagnulo S., Monier R., Khan S., Kochukhov O., Landstreet J., Wade G., Weiss W., 2007, A\&A, 476, 911
Fossati L., Bagnulo S., Landstreet J., Wade G., Kochukhov O., Monier R., Weiss W., Gebran M., 2008a, Contrib. Astron. Obs. Skalnate Pleso, 38, 123

Fossati L., Bagnulo S., Landstreet J., Wade G., Kochukhov O., Monier R., Weiss W., Gebran M., 2008b, A\&A, 483, 891

Fossati L., Ryabchikova T., Bagnulo S., Alecian E., Grunhut J., Kochukhov O., Wade G., 2009, A\&A, 503, 945

Fossati L., Mochnacki S., Landstreet J., Weiss W., 2010, A\&A, 510, A8

Fossati L., Folsom C. P., Bagnulo S., Grunhut J. H., Kochukhov O., Landstreet J. D., Paladini C., Wade G. A., 2011a, MNRAS, 413, 1132

Fossati L., Ryabchikova T., Shulyak D. V., Haswell C. A., Elmasli A., Pandey C. P., Barnes T. G., Zwintz K., 2011b, MNRAS, 417, 495

Gebran M., Monier R., 2008, A\&A, 483, 567

Gebran M., Monier R., Richard O., 2008, A\&A, 479, 189

Gebran M., Vick M., Monier R., Fossati L., 2010, A\&A, 523, 71

Glazunova L. V., Yushchenko A. V., Tsymbal V. V., Mkrtichian D. E., Lee J. J., Kang Y. W., Valyavin G. G., Lee B.-C., 2008, AJ, 136, 1736

González-García B. M., Zapatero Osorio M. R., Béjar V. J. S., Bihain G., Barrado Y Navascués D., Caballero J. A., Morales-Calderón M., 2006, A\&A, 460, 799

Grassitelli L., Fossati L., Langer N., Miglio A., Istrate A. G., Sanyal D., 2015, A\&A, 584, L2

Gray D., 2005, The Observation and Analysis of Stellar Photospheres. Cambridge Univ. Press, Cambridge

Henden A. A., Templeton M., Terrell D., Smith T. C., Levine S., Welch D., 2016, VizieR Online Data Catalog, 2336

Herbst W., 1977, AJ, 82, 902

Hubeny I., Lanz T., 1995, ApJ, 439, 875

Hubeny I., Hummer D. G., Lanz T., 1994, A\&A, 282, 151

Hui A. K., Armstrong B. H., Wray A. A., 1978, J. Quant. Spectrosc. Radiat. Transfer, 19, 509

Hummer D. G., Mihalas D., 1988, ApJ, 331, 794

Kharchenko N. V., Piskunov A. E., Schilbach E., Röser S., Scholz R.-D., 2013, A\&A, 558, A53

Kılıçoğlu T., Monier R., Richer J., Fossati L., Albayrak B., 2016, AJ, 151, 49

Kochukhov O., Makaganiuk V., Piskunov N., 2010, A\&A, 524, A5

Kurucz R., 1993a, Opacities for Stellar Atmospheres: [+0.0],[+0.5],[+1.0]. Kurucz CD-ROM No. 2. Smithsonian Astrophysical Observatory, Cambridge, MA

Kurucz R., 1993b, ATLAS9 Stellar Atmosphere Programs and $2 \mathrm{~km} / \mathrm{s}$ Grid. Kurucz CD-ROM No. 13. Smithsonian Astrophysical Observatory, Cambridge, MA

Kurucz R. L., 2005, Mem. Soc. Astron. Ital. Suppl., 8, 14

Landstreet J. D., 2004, in Zverko J., Ziznovsky J., Adelman S. J., Weiss W. W., eds, Proc. IAU Symp. 224, The A-Star Puzzle. Cambridge Univ. Press, Cambridge, p. 423

Langer N., Kudritzki R. P., 2014, A\&A, 564, A52

Levenberg K., 1944, Q. Appl. Math., 2, 164

MacQueen J., 1967, in Le Cam L. M., Neyman J., eds, Proceedings of the Fifth Berkeley Symposium on Mathematical Statistics and Probability, Volume 1: Statistics. Univ. California Press, Berkeley, CA, p. 281

Marquardt D. W., 1963, J. Soc. Ind. Appl. Math., 11, 431

Michaud G., 1970, ApJ, 160, 641

Moffat A., Vogt N., 1975, A\&AS, 20, 155

Pasquini L. et al., 2002, The Messenger, 110, 1

Piskunov N. E., Kupka F., Ryabchikova T. A., Weiss W. W., Jeffery C. S., 1995, A\&AS, 112, 525

Prsa A. et al., 2016, AJ, 152, 41

Rees D. E., Durrant C. J., Murphy G. A., 1989, ApJ, 339, 1093

Robichon N., Arenou F., Mermilliod J.-C., Turon C., 1999, A\&A, 345, 471

Roeser S., Demleitner M., Schilbach E., 2010, AJ, 139, 2440

Sanders W. L., 1971, A\&A, 14, 226

Seaton M. J., 1990, BAAS, 22, 844 
Skrutskie M. F. et al., 2006, AJ, 131, 1163

Stift M. J., 1985, MNRAS, 217, 55

Stift M., 2000, Peculiar Newsl., 27, 33

Stift M., Leone F., Cowley C., 2012, MNRAS, 419, 2912

Stütz C., Bagnulo S., Jehin E., Ledoux C., Cabanac R., Melo C., Smoker J. V., 2006, A\&A, 451, 285

Tang J., Bressan A., Rosenfield P., Slemer A., Marigo P., Girardi L., Bianchi L., 2014, MNRAS, 445, 4287
Villanova S., Carraro G., Saviane L., 2009, A\&A, 504, 845

Vogt S. S., Penrod G. D., Hatzes A. P., 1987, ApJ, 321, 496

Zacharias N., Urban S. E., Zacharias M. I., Wycoff G. L., Hall D. M., Monet D. G., Rafferty T. J., 2005, AJ, 127, 3043

This paper has been typeset from a $\mathrm{T}_{\mathrm{E}} \mathrm{X} / \mathrm{LT} \mathrm{E} \mathrm{X}$ file prepared by the author. 